\title{
Minimum essentiel et degrés d'obstruction des translatés de sous-tores
}

\author{
par \\ Patrice Philippon (Paris) et Martín Sombra (Barcelona)
}

\section{$\grave{A}$ Wolfgang Schmidt}

1. Introduction et résultats. Du point de vue de la géométrie diophantienne, les translatés de sous-tores du tore multiplicatif $\mathbb{G}_{m}^{N}:=\left(\overline{\mathbb{Q}}^{\times}\right)^{N}$ se trouvent à la croisée des problèmes de Lehmer et de Bogomolov généralisés. En effet, on sait que lorsqu'elles ne sont pas de torsion, les minorations uniformes pour la hauteur normalisée de ces variétés sont de nature arithmétique, dépendant essentiellement du corps de définition de la variété. Au contraire, pour les sous-variétés qui ne sont pas des translatés de sous-tores, on dispose de minorations ne dépendant que de leur géométrie (voir les conjectures 1.1 et 1.2 ci-dessous, voir aussi [DP99, ADa01, ADa03, Dav03]).

Le degré d'une variété n'est pas le bon invariant pour l'étude des minorations de la hauteur, il faut considérer plutôt son degré d'obstruction. Soient $X \subset Y$ des ensembles algébriques équidimensionnels de $\mathbb{P}^{N}$ définis sur un corps algébriquement clos $\mathbb{k}$ et $K$ un sous-corps de $\mathbb{k}$. Le degré d'obstruction de $X$ dans $Y$ relatif $\grave{a} K$ est

$$
\omega_{K}(X ; Y):=\min \left\{\operatorname{deg}(W): W \in \operatorname{Div}_{K}(Y), W \supset X\right\},
$$

c'est-à-dire le plus petit degré d'un diviseur $W$ de $Y$ défini sur $K$ et contenant $X$, vu comme un cycle de $\mathbb{P}^{N}$. On convient de poser $\omega_{K}(X ; Y):=\infty$ lorsque qu'aucun diviseur de $Y$ défini sur $K$ ne contient $X$.

La plus petite hauteur d'un sous-ensemble de points algébriques Zariski dense dans une variété est quantifiée par la notion de minimum essentiel. Soit $X \subset \mathbb{P}^{N}$ une variété quasi-projective quelconque définie sur $\overline{\mathbb{Q}}$, et pour

2000 Mathematics Subject Classification: Primary 11G50; Secondary 14G40, 14M25.

Key words and phrases: normalized height, multiplicative torus, essential minimum, obstruction degree, Lehmer's problem.

M. Sombra a été financé par le Programme Ramón y Cajal du Ministerio de Educación y Ciencia, Espagne. 
$\theta \geq 0$ posons $X(\theta)$ l'ensemble des points algébriques de $X$ de hauteur de Weil (ou hauteur normalisée) $\widehat{h}$ majorée par $\theta$. Le minimum essentiel de $\widehat{h}$ sur $X$ est

$$
\widehat{\mu}^{\text {ess }}(X):=\inf \{\theta: X(\theta) \text { est Zariski dense }\} .
$$

Aussi, on désigne par $\widehat{\mu}^{\text {abs }}(X)$ le minimum absolu de $\widehat{h}$ sur $X$, c'est-à-dire l'infimum des hauteurs de points algébriques de $X$.

On fixera la compactification équivariante de $\mathbb{G}_{m}^{N}$ donnée par l'inclusion standard $\iota: \mathbb{G}_{m}^{N} \hookrightarrow \mathbb{P}^{N},\left(t_{1}, \ldots, t_{N}\right) \mapsto\left(1: t_{1}: \cdots: t_{N}\right)$. Ceci permet de transporter les notions de hauteur, minimum essentiel, etc., aux sous-objets de $\mathbb{G}_{m}^{N}$ en considérant leur adhérence de Zariski dans $\mathbb{P}^{N}$.

Un sous-tore $T \subset \mathbb{G}_{m}^{N}$ est un sous-groupe algébrique isomorphe à un $\mathbb{G}_{m}^{n}$. Rappelons que tous les sous-groupes algébriques connexes de $\mathbb{G}_{m}^{N}$ sont de cette forme, et qu'en général les sous-groupes algébriques de $\mathbb{G}_{m}^{N}$ sont les produits $F \cdot T$ d'un groupe fini $F$ par un sous-tore $T$. Nous notons $\boldsymbol{\mu}^{\infty}$ le sous-groupe de $\mathbb{G}_{m}$ des racines de l'unité. Une variété de torsion $U \subset \mathbb{G}_{m}^{N}$ est le translaté d'un sous-tore par un point de torsion, c'est-à-dire un point à coordonnées dans $\boldsymbol{\mu}^{\infty}$. Pour un ensemble algébrique équidimensionnel $X \subset$ $\mathbb{G}_{m}^{N}$ on notera $U_{X}$ la plus petite réunion de variétés de torsion contenant $X$. Dans le cas où $X$ est irréductible sur $\overline{\mathbb{Q}}$, on vérifie que $U_{X}$ est également irréductible, donc une variété de torsion, car la classe des variétés de torsion contenant $X$ est fermée par intersection.

Notre motivation de départ est la minoration conjecturale suivante pour la hauteur des points Zariski dense dans une variété. Elle est proposée par Amoroso et David, en s'inspirant de divers résultats sur les problème de Lehmer et de Bogomolov généralisés; c'est une réécriture de [ADa03, Conj. 1.10] (voir §2) :

Conjecture 1.1. Il existe un réel $c(N)>0$ tel que pour toute variété $X \subset \mathbb{G}_{m}^{N}(\overline{\mathbb{Q}})$ et $U_{X}^{\mathbb{Q}}$ la plus petite réunion de variétés de torsion contenant $X$ qui soit définie sur $\mathbb{Q}$, on ait $\left(^{1}\right)$

$$
\widehat{\mu}^{\operatorname{ess}}(X) \geq c(N) \frac{\operatorname{deg}\left(U_{X}^{\mathbb{Q}}\right)}{\omega_{\mathbb{Q}}\left(X ; U_{X}^{\mathbb{Q}}\right)} .
$$

On notera que $U_{X}^{\mathbb{Q}}$ n'est autre que la réunion des images de $U_{X}$ sous l'action du groupe de Galois de $\overline{\mathbb{Q}}$ sur $\mathbb{Q}$. Cette conjecture est équivalente, à la valeur de la constante $c(N)$ près, à son analogue sur la clôture abélienne $\mathbb{Q}^{\mathrm{ab}}$ de $\mathbb{Q}$ (cf. proposition 2.5) :

(1.abélien)

$$
\widehat{\mu}^{\mathrm{ess}}(X) \geq c^{\prime}(N) \frac{\operatorname{deg}\left(U_{X}\right)}{\omega_{\mathbb{Q}^{\mathrm{ab}}}\left(X ; U_{X}\right)} .
$$

$\left({ }^{1}\right) \operatorname{Si} \operatorname{dim}(X)=\operatorname{dim}\left(U_{X}^{\mathbb{Q}}\right)$ on a $\omega_{\mathbb{Q}}\left(X ; U_{X}^{\mathbb{Q}}\right)=\infty$ et $\operatorname{deg}\left(U_{X}^{\mathbb{Q}}\right) / \omega_{\mathbb{Q}}\left(X ; U_{X}^{\mathbb{Q}}\right)=0$. 
On remarquera la cohérence de l'énoncé ci-dessus avec la conjecture 1.1, car $U_{X}=U_{X}^{\mathbb{Q}^{\mathrm{ab}}}$ par le lemme 2.3.

La conjecture 1.1 est surtout intéressante pour les translatés de sous-tores par des points d'ordre infini, car pour les autres variétés on s'attend à une minoration à caractère géométrique, ne dépendant pas du corps de définition de la variété ( « problème de Bogomolov effectif ») :

Conjecture 1.2 ([ADa03, Conj. 1.2]). Il existe un réel $c(N)>0$ tel que pour toute variété $X \subset \mathbb{G}_{m}^{N}(\overline{\mathbb{Q}})$ et $B_{X}$ le plus petit translaté de sous-tore contenant $X$, on ait

$$
\widehat{\mu}^{\text {ess }}(X) \geq c(N) \frac{\operatorname{deg}\left(B_{X}\right)}{\omega_{\overline{\mathbb{Q}}}\left(X ; B_{X}\right)} .
$$

Notons toutefois que cette minoration, si elle est de nature purement géométrique, n'en est pas toujours meilleure que celle de la conjecture 1.1 (voir remarque 4.3).

Pour un sous-corps $K \subset \overline{\mathbb{Q}}$ on pose

$$
m(K):=\inf \left\{\widehat{h}(\alpha): \alpha \in K^{\times} \backslash \boldsymbol{\mu}^{\infty}\right\} .
$$

Dans ce texte nous présentons la minoration suivante pour la hauteur des points d'un translaté de sous-tore par un point d'ordre infini :

ThÉORÈme 1.3. Soit $X \subset \mathbb{G}_{m}^{N}$ un translaté de sous-tore, défini sur un corps de nombres $K$. Alors

$$
\widehat{\mu}^{\mathrm{abs}}(X) \geq c_{1}(N) m(K) \frac{\operatorname{deg}\left(U_{X}\right)}{\omega_{\overline{\mathbb{Q}}}\left(X ; U_{X}\right)} \quad \text { avec } \quad c_{1}(N):=N^{-3 / 2} 2^{-N} .
$$

Ce théorème concerne le minimum absolu de $\widehat{h}$ sur $X$; on se reportera à sa preuve au $\S 4$ pour une valeur plus précise de la constante. Or, pour les translatés de sous-tores les minimums absolu et essentiel coïncident et donc cette minoration peut de façon équivalente se reformuler en termes de $\widehat{\mu}^{\text {ess }}$. Notons aussi qu'un translaté de sous-tore $X$ est défini sur $K$ si et seulement si c'est le translaté par un point défini sur $K$, c'est-à-dire si et seulement s'il contient un point $K$-rationnel (voir §3). De plus, la variété de torsion minimale $U_{X}$ est automatiquement définie sur $K$ elle aussi (voir lemme 2.3).

Dans la suite de ce texte nous allons voir que le théorème 1.3 entretient une relation ambigüe avec la conjecture 1.1. L'ambigüité tient pour l'essentiel dans le comportement de l'invariant $m(K)$, qui ne se laisse pas facilement contrôler en fonction du degré du corps de nombres $K$. D'une part, le théorème 1.3 n'est a priori pas conséquence de la conjecture 1.1, car il entraîne en particulier le corollaire 1.4 ci-dessous qui donne une minoration plus forte que celle de cette conjecture. D'autre part, l'exemple 3.2 montre que le théorème 1.3 ne peut entrainer la conjecture 1.1. Ainsi, en combinant le théorème 1.3 avec le problème de Lehmer (que nous rappellons ci-dessous) 
on n'aboutit qu'à une conséquence affaiblie de la conjecture 1.1, où l'on y remplace $\operatorname{deg}\left(U_{X}^{\mathbb{Q}}\right) / \omega_{\mathbb{Q}}\left(X ; U_{X}^{\mathbb{Q}}\right) \operatorname{par} \operatorname{deg}\left(U_{X}\right) /[K: \mathbb{Q}] \omega_{\overline{\mathbb{Q}}}\left(X ; U_{X}\right)(\operatorname{voir} \S 2.2)$.

Le problème de Lehmer classique consiste à montrer l'existence d'une constante $c>0$ telle que $m(K) \geq c[K: \mathbb{Q}]^{-1}$ pour tout corps de nombres $K$. Le meilleur résultat connu dans cette direction reste celui de Dobrowolski [Dob79]

$$
m(K) \geq \frac{c}{[K: \mathbb{Q}]}\left(\frac{\log \log (3[K: \mathbb{Q}])}{\log (2[K: \mathbb{Q}])}\right)^{3} .
$$

De fait, Amoroso et David ont démontré les conjectures 1.1 et 1.2 à des facteurs logarithmiques près (lorsque $U_{X}$ et $B_{X}$ sont égaux à $\mathbb{G}_{m}^{N}$ respectivement) [ADa01, ADa03], notamment par une extension non triviale de l'approche de Dobrowolski pour la conjecture 1.1.

Par ailleurs, on sait par des travaux de Schinzel [Sch73] que lorsque $K$ est un corps totalement réel ou CM (c'est-à-dire une extension imaginaire quadratique d'un corps totalement réel) et $\alpha \in K^{\times}$satisfait $|\alpha| \neq 1$, alors

$$
\widehat{h}(\alpha) \geq \frac{1}{2} \log \left(\frac{1+\sqrt{5}}{2}\right),
$$

avec égalité si et seulement si $\alpha=(1 \pm \sqrt{5}) / 2$. D'après [AN07], la condition $|\alpha| \neq 1$ ne peut être éliminée $\left({ }^{2}\right)$, notons cependant que lorsque $\alpha$ est entier mais pas une racine de l'unité, elle est satisfaite par au moins un conjugué de $\alpha$, et lorsque $K$ est totalement réel, elle est satisfaite par tout $\alpha \in K^{\times} \backslash \boldsymbol{\mu}^{\infty}$. Amoroso et Dvornicich ont montré [ADv00] qu'à l'instar du cas totalement réel, dans une extension abélienne $K$ de $\mathbb{Q}$ on a une minoration uniforme pour la hauteur des nombres algébriques non nuls qui ne sont pas des racines de l'unité :

$$
m(K) \geq \frac{\log (5)}{12} .
$$

En combinant le théorème 1.3 avec les inégalités (2) et (3) on obtient la généralisation suivante (à la constante près) des résultats de [Sch73] et [ADv00] :

Corollaire 1.4. Soit $X \subset \mathbb{G}_{m}^{N}$ un translaté de sous-tore défini sur un corps $K$ totalement réel ou une extension abélienne de $\mathbb{Q}$. Alors

$$
\widehat{\mu}^{\text {abs }}(X) \geq c_{2}(N) \frac{\operatorname{deg}\left(U_{X}\right)}{\omega_{\overline{\mathbb{Q}}}\left(X ; U_{X}\right)} \quad \text { avec } \quad c_{2}(N):=\frac{\log (5)}{12} c_{1}(N) .
$$

$\left({ }^{2}\right)$ Comme nous l'a signalé Amoroso, ceci se déduit également directement de la proposition 1 de loc. cit. Par exemple, pour tout $d \in \mathbb{N}$ le corps $\mathbb{Q}\left(\alpha^{1 / d}\right)$ avec $\alpha=$ $(\sqrt{2}-i) /(\sqrt{2}+i)$ est un corps CM par cette proposition. On vérifie que $h\left(\alpha^{1 / d}\right)$ est arbitrairement petit lorsque $d$ tends vers l'infini, toutefois $\alpha^{1 / d}$ n'est pas une racine de l'unité mais tous ses conjugués sont de module 1 . 
Plus généralement, si $K$ est un corps $C M$ notons $G_{X}$ la plus petite variété de torsion contenant $X \cdot \bar{X}$ (ici $\bar{X}$ désigne l'image de $X$ sous l'action de la conjugaison complexe). Alors

$$
\widehat{\mu}^{\mathrm{abs}}(X) \geq c_{2}(N) \frac{\operatorname{deg}\left(G_{X}\right)}{\omega_{\overline{\mathbb{Q}}}\left(X \cdot \bar{X} ; G_{X}\right)} .
$$

On montre que, dans la situation de ce corollaire et pour un corps CM, la variété de torsion $G_{X}$ est en fait un sous-tore (lemme 4.2). La minoration du corollaire 1.4 pour $K$ un corps totalement réel se compare favorablement à celle de la conjecture 1.1, grâce au lemme 2.4. Dans le cas d'une extension abélienne de $\mathbb{Q}$, elle est par ailleurs conséquence de la conjecture 1.1 suivant un argument de David (voir proposition 2.5).

La preuve de ces résultats s'appuie sur une étude des degrés d'obstruction des translatés de sous-tores dans les tores. Remarquons que si $X \subset Y$ sont des variétés définies sur un sous-corps $K \subset \overline{\mathbb{Q}}$, il n'existe pas nécessairement de diviseur défini sur $K$ réalisant $\omega_{\overline{\mathbb{Q}}}(X ; Y)$ et on n'a donc pas en général $\omega_{K}(X ; Y)=\omega_{\overline{\mathbb{Q}}}(X ; Y)$ comme le montrent des exemples au $\S 2$. C'est toutefois le cas lorsque $Y$ est un translaté de sous-tore (voir corollaire 2.2).

On démontre que lorsque $X$ et $Y$ sont des translatés de sous-tores de dimensions respectives $n$ et $p$, le degré d'obstruction relatif à $\overline{\mathbb{Q}}$ se réalise par une équation binomiale, et qu'il est comparable au premier minimum d'un certain réseau (proposition 3.3). Comme conséquence, on peut le majorer grâce au premier théorème de Minkowski par (corollaire 3.5)

$$
\omega_{\overline{\mathbb{Q}}}(X ; Y) \leq 4^{N} N^{3 / 2} \operatorname{deg}(X)^{1 /(p-n)} \operatorname{deg}(Y)^{1-1 /(p-n)} .
$$

L'inégalité plus précise du lemme 3.4 jointe au théorème 1.3 entraîne :

Corollaire 1.5. Soit $X \subset \mathbb{G}_{m}^{N}$ un translaté de sous-tore par un point d'ordre infini et défini sur un corps de nombres $K$. Alors

$$
\widehat{\mu}^{\mathrm{abs}}(X) \geq 2^{-N} N^{-2} m(K)\left(\frac{\operatorname{deg}\left(U_{X}\right)}{\operatorname{deg}(X)}\right)^{1 / \operatorname{codim}_{U_{X}}(X)} .
$$

Cette minoration peut alternativement se regarder comme une majoration pour le degré de la variété de torsion minimale $U_{X}$. Si $X$ est réduite à un point $\alpha \in\left(K^{\times}\right)^{N} \backslash\left(\boldsymbol{\mu}^{\infty}\right)^{N}$, alors

$$
\widehat{h}(\alpha) \geq 2^{-N} N^{-2} m(K) \operatorname{deg}\left(U_{\alpha}\right)^{1 / \operatorname{dim}\left(U_{\alpha}\right)} .
$$

On retrouve ainsi la minoration $(*)$ de [Ber95] pour le cas des tores déployés, avec de plus une dépendance précise dans le corps $K$ (comparer avec [Ber95, Cor. 1]). Ce type de minoration a des applications au calcul du module des relations de dépendance multiplicative des nombres (voir aussi à ce propos [Ber97]). 
Finalement, on étudie l'analogue fonctionnel de la conjecture 1.1. Soit $\mathbb{k}$ un corps algébriquement clos; on s'intéressera à la hauteur des points dans une variété $X \subset \mathbb{P}^{N}(\overline{\mathbb{k}(t)})$. Le rôle des racines de l'unité est joué par les éléments de $\mathbb{k}$, et celui des variétés de torsion par les variétés définies sur $\mathbb{k}$. Notons $U_{X}$ la plus petite variété définie sur $\mathbb{k}$ et contenant $X$. On montre que si $X$ elle même n'est pas définie sur $\mathbb{k}$, alors elle est de codimension 1 dans $U_{X}$, et que son minimum essentiel est minoré par (proposition 5.1)

$$
\mu^{\mathrm{ess}}(X) \geq \frac{1}{d+1} \frac{\operatorname{deg}\left(U_{X}\right)}{\omega_{\mathbb{k}(t)}\left(X ; U_{X}\right)} .
$$

La démonstration se base sur la théorie de l'intersection multiprojective élémentaire.

Le texte est organisé de la façon suivante : aux $\S 2$ et $\S 3$ on étudie le degré d'obstruction dans les sous-tores et on établit sa relation avec le premier minimum d'un certain réseau. Nous montrons le théorème 1.3 et ses corollaires au $\S 4$, les démonstrations n'utilisent que les arguments de nature géométrique développés aux paragraphes précédents. Au $§ 5$ nous étudions l'analogue fonctionnel de la conjecture 1.1.

Remerciements. Nous remercions Sinnou David pour son aide au cours de la génèse de ce travail, ainsi que Francesco Amoroso pour ses remarques sur une première version de ce texte.

2. Degrés d'obstruction dans les tores. Le but de ce paragraphe est de décrire en termes combinatoires les degrés des diviseurs des translatés de sous-tores. Pour cette étude on peut se ramener à supposer, quitte à translater la situation, que le translaté de sous-tore en question est en fait un sous-tore.

2.1. La situation géométrique. Soit $\mathbb{k}$ un corps algébriquement clos. On pose $\mathbb{G}_{m}^{N}(\mathbb{k}):=\left(\mathbb{k}^{\times}\right)^{N}$ le groupe multiplicatif et $\mathbb{P}^{N}(\mathbb{k})$ l'espace projectif sur $\mathbb{k}$; on omettra la mention au corps lorsque celui-ci sera clair dans le contexte. Une variété sera une sous-variété réduite et irréductible de $\mathbb{P}^{N}(\mathbb{k})$ ou de $\mathbb{G}_{m}^{N}(\mathbb{k})$. Pour un sous-corps $K$ de $\mathbb{k}$, une $K$-variété sera un sous-ensemble algébrique de $\mathbb{P}^{N}(\mathbb{k})$ ou de $\mathbb{G}_{m}^{N}(\mathbb{k})$, défini sur $K$, réduit et $K$-irréductible. Pour une variété $X$ on désigne par $I(X)$ son idéal de définition dans $\mathbb{k}\left[x_{0}, \ldots, x_{N}\right]$ ou dans $\mathbb{k}\left[x_{1}^{ \pm 1}, \ldots, x_{N}^{ \pm 1}\right]$, suivant que $X \subset \mathbb{P}^{N}$ ou $X \subset \mathbb{G}_{m}^{N}$.

Commençons par décrire les sous-tores à l'aide de paramétrisations. Soit $\mathcal{B}=\left(b_{1}, \ldots, b_{N}\right) \in\left(\mathbb{Z}^{p}\right)^{N} ;$ le sous-tore associé $T_{\mathcal{B}} \subset \mathbb{G}_{m}^{N}(\mathbb{k})$ est par définition l'image de l'application monomiale (pour $t \in \mathbb{G}_{m}^{p}(\mathbb{k})$ et $b \in \mathbb{Z}^{p}$ on note $\left.t^{b}:=t_{1}^{b_{1}} \ldots t_{p}^{b_{p}}\right)$

$$
\varphi_{\mathcal{B}}: \mathbb{G}_{m}^{p}(\mathbb{k}) \rightarrow \mathbb{G}_{m}^{N}(\mathbb{k}), \quad t \mapsto\left(t^{b_{1}}, \ldots, t^{b_{N}}\right) .
$$


On supposera dans la suite que le $\mathbb{Z}$-module $L_{\mathcal{B}}:=\mathbb{Z} b_{1}+\cdots+\mathbb{Z} b_{N}$ coïncide avec $\mathbb{Z}^{p}$. Ceci équivaut à ce que $\varphi_{\mathcal{B}}$ soit un isomorphisme entre $\mathbb{G}_{m}^{p}$ et $T_{\mathcal{B}}$, et entraîne $\operatorname{donc} \operatorname{dim}\left(T_{\mathcal{B}}\right)=p$. Pour $\beta=\left(\beta_{1}, \ldots, \beta_{N}\right) \in \mathbb{G}_{m}^{N}$ on note $\beta T_{\mathcal{B}}$ le translaté de $T_{\mathcal{B}}$ par le point $\beta$. Dans les notations de [PS04], $T_{\mathcal{B}}$ (resp. $\beta T_{\mathcal{B}}$ ) est l'ouvert principal de la variété torique $X_{\mathcal{B}^{\prime}} \subset \mathbb{P}^{N}$ (resp. $X_{\mathcal{B}^{\prime}, \beta^{\prime}}$ ) pour $\mathcal{B}^{\prime}:=\left(\mathbf{0}, b_{1}, \ldots, b_{N}\right) \in\left(\mathbb{Z}^{p}\right)^{N+1}$ et $\beta^{\prime}:=\left(1, \beta_{1}, \ldots, \beta_{N}\right) \in\left(\mathbb{k}^{\times}\right)^{N+1}$.

Soit $Q_{\mathcal{B}} \subset \mathbb{R}^{p}$ l'enveloppe convexe des points $\mathbf{0}, b_{1}, \ldots, b_{N}$ et introduisons aussi l'application linéaire

$$
M_{\mathcal{B}}: \mathbb{R}^{N} \rightarrow \mathbb{R}^{p}, \quad \lambda \mapsto \lambda_{1} b_{1}+\cdots+\lambda_{N} b_{N}
$$

Pour un polynôme de Laurent $f \in \mathbb{k}\left[x_{1}^{ \pm 1}, \ldots, x_{N}^{ \pm 1}\right]$ on note $\mathrm{N}(f) \subset \mathbb{R}^{N}$ son polytope de Newton, enveloppe convexe des exposants des monômes apparaissant effectivement dans l'écriture de $f$. Soit $\varphi_{\mathcal{B}}^{*}(f):=f \circ \varphi_{\mathcal{B}} \in$ $\mathbb{k}\left[t_{1}^{ \pm 1}, \ldots, t_{p}^{ \pm 1}\right] ;$ alors $\mathrm{N}\left(\varphi_{\mathcal{B}}^{*}(f)\right)=M_{\mathcal{B}}(\mathrm{N}(f))$.

Le volume mixte (ou multi-volume) $\operatorname{MV}\left(Q_{1}, \ldots, Q_{p}\right.$ ) d'une famille d'ensembles convexes $Q_{1}, \ldots, Q_{p} \subset \mathbb{R}^{p}$ généralise la notion de volume euclidien, noté $\mathrm{Vol}_{n}$, d'un convexe; il est défini par la formule

$$
\operatorname{MV}\left(Q_{1}, \ldots, Q_{p}\right):=\sum_{j=1}^{p}(-1)^{p-j} \sum_{1 \leq i_{1}<\cdots<i_{j} \leq p} \operatorname{Vol}_{n}\left(Q_{i_{1}}+\cdots+Q_{i_{j}}\right),
$$

oú l'on désigne par + la somme de Minkowski des convexes. En particulier, si $Q_{1}=\cdots=Q_{p}=Q$ on a $\operatorname{MV}(Q, \ldots, Q)=n$ ! $\operatorname{Vol}_{n}(Q)$. Le volume mixte est symétrique, linéaire en chaque variable $Q_{i}$ par rapport à la somme de Minkowski et monotone par rapport à l'inclusion (voir par exemple [Ewa96]).

Le résultat suivant donne une description combinatoire du degré du diviseur découpé par $f$ sur $T_{\mathcal{B}}$; on y note $\cdot$ le produit d'intersection dans $\mathbb{G}_{m}^{N}(\mathbb{k})$ :

Proposition 2.1. Soit $\mathcal{B} \in\left(\mathbb{Z}^{p}\right)^{N+1}$ et $f \in \mathbb{k}\left[t_{1}^{ \pm 1}, \ldots, t_{N}^{ \pm 1}\right] \backslash I\left(T_{\mathcal{B}}\right)$. On suppose $L_{\mathcal{B}}=\mathbb{Z}^{p}$. Alors

$$
\operatorname{deg}\left(\operatorname{div}(f) \cdot T_{\mathcal{B}}\right)=\operatorname{MV}(M_{\mathcal{B}}(\mathrm{N}(f)), \underbrace{Q_{\mathcal{B}}, \ldots, Q_{\mathcal{B}}}_{p-1 \text { fois }}),
$$

où - désigne le produit d'intersection d'un diviseur avec un cycle. Plus généralement, soit $Y=\bigcup_{i=1}^{D} \beta_{i} T_{\mathcal{B}}$ une réunion de $D$ translatés de $T_{\mathcal{B}}$. Alors

$$
\operatorname{deg}(\operatorname{div}(f) \cdot Y)=D \cdot \operatorname{MV}\left(M_{\mathcal{B}}(\mathrm{N}(f)), Q_{\mathcal{B}}, \ldots, Q_{\mathcal{B}}\right) .
$$

Démonstration. Posons $W:=\operatorname{div}(f) \cdot T_{\mathcal{B}} \in \operatorname{Div}\left(T_{\mathcal{B}}\right)$, vu comme un cycle de dimension $p-1$ de $\mathbb{P}^{N}$. On a $\operatorname{deg}(W)=\operatorname{Card}\left(W \cap Z\left(\ell_{1}, \ldots, \ell_{p-1}\right)\right)$ pour $\ell_{i} \in \mathbb{k}\left[x_{1}, \ldots, x_{N}\right]$ des formes linéaires génériques et donc

$$
\operatorname{deg}(W)=\operatorname{Card}\left(\varphi_{\mathcal{B}}^{*}(f) \cap \varphi_{\mathcal{B}}^{*}\left(\ell_{1}\right) \cap \cdots \cap \varphi_{\mathcal{B}}^{*}\left(\ell_{p-1}\right)\right)
$$


car $\varphi_{\mathcal{B}}: \mathbb{G}_{m}^{p} \rightarrow T_{\mathcal{B}}$ est une bijection. Le théorème de Kushnirenko-Bernshteĭn [Bern75] entraîne alors la majoration

$$
\begin{aligned}
\operatorname{deg}(W) & \leq \operatorname{MV}\left(\mathrm{N}\left(\varphi_{\mathcal{B}}^{*}(f)\right), \mathrm{N}\left(\varphi_{\mathcal{B}}^{*}\left(\ell_{1}\right)\right), \ldots, \mathrm{N}\left(\varphi_{\mathcal{B}}^{*}\left(\ell_{p-1}\right)\right)\right) \\
& =\operatorname{MV}\left(M_{\mathcal{B}}(\mathrm{N}(f)), Q_{\mathcal{B}}, \ldots, Q_{\mathcal{B}}\right)
\end{aligned}
$$

$\operatorname{car} \mathrm{N}\left(\varphi_{\mathcal{B}}^{*}\left(\ell_{i}\right)\right)=Q_{\mathcal{B}}$.

Pour $\tau \in \mathbb{R}^{p}$ et $g \in \mathbb{k}\left[t_{1}^{ \pm 1}, \ldots, t_{p}^{ \pm 1}\right]$ notons $\operatorname{init}_{\tau}(g) \in \mathbb{k}\left[t_{1}^{ \pm 1}, \ldots, t_{p}^{ \pm 1}\right]$ la partie initiale de $g$ relative au poids $\tau$, c'est-à-dire la partie de $g$ de poids maximal dans la direction de $\tau$ (i.e. la somme des monômes de $g$ dont les exposants maximisent le produit scalaire avec $\tau$ ). Bernshteĭn démontre aussi que la majoration (5) est une égalité si et seulement si pour tout $\tau \in \mathbb{R}^{p}$ le système

$$
\operatorname{init}_{\tau}\left(\varphi_{\mathcal{B}}^{*}(f)\right)=0, \quad \operatorname{init}_{\tau}\left(\varphi_{\mathcal{B}}^{*}\left(\ell_{1}\right)\right)=0, \quad \ldots, \quad \operatorname{init}_{\tau}\left(\varphi_{\mathcal{B}}^{*}\left(\ell_{p-1}\right)\right)=0
$$

n'a pas de solution dans $\mathbb{G}_{m}^{p}$. Or, $\operatorname{init}_{\tau}\left(\varphi_{\mathcal{B}}^{*}(f)\right)$ est toujours un polynôme de Laurent non nul puisque $f \neq 0$, et donc cette condition est satisfaite par des formes linéaires $\ell_{i}$ génériques, ce qui démontre le résultat.

Pour vérifier la dernière égalité de la proposition, par additivité du degré il suffit de vérifier

$$
\operatorname{deg}\left(\operatorname{div}(f) \cdot T_{\mathcal{B}}\right)=\operatorname{deg}\left(\operatorname{div}(f) \cdot\left(\beta T_{\mathcal{B}}\right)\right)
$$

pour tout $\beta \in \mathbb{G}_{m}^{N}(\mathbb{k})$. On a $\operatorname{div}(f) \cdot\left(\beta T_{\mathcal{B}}\right)=\beta \cdot\left(\operatorname{div}\left(\left(\beta^{-1}\right)^{*}(f)\right) \cdot T_{\mathcal{B}}\right)$. Or le degré est invariant par translation et $\varphi_{\mathcal{B}}^{*} \circ\left(\beta^{-1}\right)^{*}(f)$ et $\varphi_{\mathcal{B}}^{*}(f)$ ont le même support, l'identité cherchée résulte donc de la formule précédemment établie.

Corollaire 2.2. Soit $K \subset \mathbb{k}$ un sous-corps et $X \subset T_{\mathcal{B}}$ une $K$-variété. Alors, dans les notations de la proposition 2.1,

$$
\omega_{K}\left(X ; T_{\mathcal{B}}\right)=\min \left\{\operatorname{MV}\left(M_{\mathcal{B}}(\mathrm{N}(f)), Q_{\mathcal{B}}, \ldots, Q_{\mathcal{B}}\right): f \in I(X) \backslash I\left(T_{\mathcal{B}}\right)\right\} .
$$

En particulier, $\omega_{K}\left(X ; T_{\mathcal{B}}\right)=\omega_{\mathbb{k}}\left(X ; T_{\mathcal{B}}\right)$.

Démonstration. L'anneau $\mathbb{k}\left[T_{\mathcal{B}}\right]$ est factoriel car il est isomorphe à $\mathbb{k}\left[t_{1}^{ \pm 1}, \ldots, t_{p}^{ \pm 1}\right]$. Il est donc principal et tout diviseur de $T_{\mathcal{B}}$ contenant $X$ est défini par une seule équation $f \in I(X) \backslash I\left(T_{\mathcal{B}}\right)$.

On écrit $f$ comme combinaison linéaire de monômes indépendants modulo $I\left(T_{\mathcal{B}}\right)$. Comme $X$ est une $K$-variété, la condition énonçant que $f$ s'annule sur $X$ s'écrit à travers d'un système linéaire défini sur $K$, on peut donc en trouver une solution non triviale $g \in K\left[x_{1}^{ \pm 1}, \ldots, x_{N}^{ \pm 1}\right]$ qui satisfait $\operatorname{Supp}(g) \subset$ $\operatorname{Supp}(f)\left(\right.$ donc $\left.M_{\mathcal{B}}(\mathrm{N}(g)) \subset M_{\mathcal{B}}(\mathrm{N}(f))\right)$ et qui définit un $K$-diviseur de $T_{\mathcal{B}}$. Il suit de la proposition 2.1 et de la monotonie du volume mixte par rapport à l'inclusion [Ewa96, Thm. 4.12] que le degré du $K$-diviseur $\operatorname{div}(g) \cdot T_{\mathcal{B}}$ est inférieur ou égal à celui $\operatorname{de} \operatorname{div}(f) \cdot T_{\mathcal{B}}$. Finalement, en considérant un 
diviseur réalisant $\omega_{\mathbb{k}}\left(X ; T_{\mathcal{B}}\right)$ on conclut $\omega_{\mathbb{k}}\left(X ; T_{\mathcal{B}}\right)=\omega_{K}\left(X ; T_{\mathcal{B}}\right)$, puis avec la proposition 2.1 on obtient l'expression de $\omega_{K}\left(X ; T_{\mathcal{B}}\right)$ mentionnée.

Lorsque $Y$ n'est pas un translaté de sous-tore, tout en restant défini sur $K$, on n'a plus nécessairement l'égalité $\omega_{K}(X ; Y)=\omega_{\mathbb{k}}(X ; Y)$, même lorsque $X$ est elle-même un translaté de sous-tore défini sur $K$. En effet, considérons $X:=\{(1: 1: 1: 1)\}$ dans la réunion $Y$ de quatre plans de $\mathbb{P}^{3}$ définie par l'équation, rationnelle sur $\mathbb{Q}$, suivante :

$$
\prod_{i, j \in\{0,1\}}\left((w-x)+(-1)^{i} \sqrt{2}(w-y)+(-1)^{j} \sqrt{3}(w-z)\right)=0 .
$$

Une droite rationnelle sur $\mathbb{Q}$ passant par le point $X$ admet une paramétrisation de la forme

$$
\mathbb{P}^{1} \rightarrow \mathbb{P}^{3}, \quad\left(t_{0}: t_{1}\right) \mapsto\left(t_{0}: a t_{1}+t_{0}: b t_{1}+t_{0}: c t_{1}+t_{0}\right),
$$

avec $a, b, c \in \mathbb{Q}$ non tous nuls. On en déduit qu'une telle droite ne peut être contenue dans $Y$, car $1, \sqrt{2}$ et $\sqrt{3}$ sont linéairement indépendants sur $\mathbb{Q}$, et donc $\omega_{\mathbb{Q}}(X ; Y) \geq 2($ en fait $=2)$, tandis que $\omega_{\overline{\mathbb{Q}}}(X ; Y)=1$ car $Y$ contient une infinité de droites (définies sur $\overline{\mathbb{Q}}$ ) passant par $X$.

On peut aussi produire un exemple où $Y$ est irréductible : soit $Y \subset \mathbb{P}^{3}$ la surface, définie sur $\mathbb{Q}$ et irréductible sur $\overline{\mathbb{Q}}$, d'équation $w z-x^{2}+2 y^{2}=0$ et $X$ un des points $(1: 0: 0: 0)$ ou $(0: 0: 0: 1)$. Cette surface contient deux droites définies sur $\mathbb{Q}(\sqrt{2})$ passant par chacun des points fixés, mais aucune droite définie sur $\mathbb{Q}$ passant par un de ces mêmes points.

2.2. Particularités arithmétiques. On se place maintenant sur $\mathbb{k}=\overline{\mathbb{Q}}$. Soit $X \subset \mathbb{G}_{m}^{N}(\overline{\mathbb{Q}})$ un ensemble algébrique équidimensionnel et $K$ un corps de nombres. Notons $U_{X}^{K}$ la plus petite réunion de variétés de torsion contenant $X$ qui soit définie sur $K$. Notons qu'une réunion de variétés de torsion est toujours définie sur $\mathbb{Q}^{\mathrm{ab}}$, la clôture abélienne de $\mathbb{Q}$, et donc $U_{X}^{K}$ est définie sur $K \cap \mathbb{Q}^{\text {ab }}$, la sous-extension abélienne maximale de $K$.

Lemme 2.3. Soit $X \subset \mathbb{G}_{m}^{N}(\overline{\mathbb{Q}})$ une variété définie sur un corps de nombres $K$. Alors $U_{X}=U_{X}^{\overline{\mathbb{Q}}}$, la plus petite variété de torsion contenant $X$, est définie sur $K \cap \mathbb{Q}^{\mathrm{ab}}$, et elle coïncide avec $U_{X}^{K}$.

Démonstration. Ceci résulte de ce que la classe des réunions de variétés de torsion (à laquelle il convient d'adjoindre la variété vide) est fermée par intersections : si $U_{X}$ est la plus petite réunion de variétés de torsion contenant $X$, alors $\sigma\left(U_{X}\right)=U_{X}$ pour tout $\sigma \in \operatorname{Gal}(\overline{\mathbb{Q}} / K)$, donc $U_{X}$ est définie sur $K$ et par suite sur $K \cap \mathbb{Q}^{\text {ab }}$.

Comme conséquence de ceci et de l'égalité

$$
\omega_{K}(X ; Y)=\omega_{K}\left(\bigcup_{\sigma \in \operatorname{Gal}(\overline{\mathbb{Q}} / K)} \sigma(X) ; Y\right),
$$


valable pour toute variété $X$ définie sur $\overline{\mathbb{Q}}$, la conjecture 1.1 peut se reformuler de la façon suivante, plus proche de la formulation originale dans [ADa03, Conj. 1.10] : si $X \subset \mathbb{G}_{m}^{N}$ est une $\mathbb{Q}$-variété et $U_{X}$ la plus petite réunion de variétés de torsion contenant $X$, alors il existe un réel $c(N)>0$ indépendant de $X$ tel que

$$
\widehat{\mu}^{\operatorname{ess}}(X) \geq c(N) \frac{\operatorname{deg}\left(U_{X}\right)}{\omega_{\mathbb{Q}}\left(X ; U_{X}\right)} .
$$

Lemme 2.4. Soit $X \subset \mathbb{G}_{m}^{N}$ une variété et $K_{1}$ le corps de définition de $U_{X}$. Alors

$$
\operatorname{deg}\left(U_{X}^{\mathbb{Q}}\right)=\left[K_{1}: \mathbb{Q}\right] \operatorname{deg}\left(U_{X}\right), \quad \omega_{\mathbb{Q}}\left(X ; U_{X}^{\mathbb{Q}}\right)=\left[K_{1}: \mathbb{Q}\right] \omega_{K_{1}}\left(X ; U_{X}\right) .
$$

En particulier, si $X$ est définie sur un corps de nombres $K$ on a

$$
\frac{1}{\left[K: K_{1}\right]} \frac{\operatorname{deg}\left(U_{X}\right)}{\omega_{\overline{\mathbb{Q}}}\left(X ; U_{X}\right)} \leq \frac{\operatorname{deg}\left(U_{X}^{\mathbb{Q}}\right)}{\omega_{\mathbb{Q}}\left(X ; U_{X}^{\mathbb{Q}}\right)}=\frac{\operatorname{deg}\left(U_{X}\right)}{\omega_{K_{1}}\left(X ; U_{X}\right)} \leq \frac{\operatorname{deg}\left(U_{X}\right)}{\omega_{\overline{\mathbb{Q}}}\left(X ; U_{X}\right)} .
$$

Démonstration. On a $U_{X}^{\mathbb{Q}}=\bigcup_{\sigma} \sigma\left(U_{X}\right)$ où $\sigma$ parcourt les $\mathbb{Q}$-plongements de $K_{1}$ dans $\overline{\mathbb{Q}}$, d'où la première égalité. Soit $E \subset U_{X}$ un $K_{1}$-diviseur réalisant $\omega_{K_{1}}\left(X ; U_{X}\right)$; alors $\bigcup_{\sigma} \sigma(E)$ est un $\mathbb{Q}$-diviseur de $U_{X}^{\mathbb{Q}}$ de degré $\leq\left[K_{1}: \mathbb{Q}\right] \omega_{K_{1}}\left(X ; U_{X}\right)$ et contenant $X$, d'où

$$
\omega_{\mathbb{Q}}\left(X ; U_{X}^{\mathbb{Q}}\right) \leq\left[K_{1}: \mathbb{Q}\right] \omega_{K_{1}}\left(X ; U_{X}\right) .
$$

Si $E^{\mathbb{Q}}$ est un $\mathbb{Q}$-diviseur de $U_{X}^{\mathbb{Q}}$ réalisant $\omega_{\mathbb{Q}}\left(X ; U_{X}^{\mathbb{Q}}\right)$ alors $E^{\mathbb{Q}} \cap U_{X}$ est un $K_{1}$-diviseur de $U_{X}$ contenant $X$ et donc de degré $\geq \omega_{K_{1}}\left(X ; U_{X}\right)$. Mais $\bigcup_{\sigma} \sigma\left(E^{\mathbb{Q}} \cap U_{X}\right)$ est contenu dans $E^{\mathbb{Q}}$ et de degré $\geq\left[K_{1}: \mathbb{Q}\right] \omega_{K_{1}}\left(X ; U_{X}\right)$, on a donc

$$
\omega_{\mathbb{Q}}\left(X ; U_{X}^{\mathbb{Q}}\right) \geq\left[K_{1}: \mathbb{Q}\right] \omega_{K_{1}}\left(X ; U_{X}\right)
$$

et la seconde égalité.

L'égalité centrale de la dernière formule de l'énoncé résulte de ce qui précède, et les inégalités extrêmes de l'encadrement

$$
\omega_{\overline{\mathbb{Q}}}\left(X ; U_{X}\right) \leq \omega_{K_{1}}\left(X ; U_{X}\right) \leq\left[K: K_{1}\right] \omega_{\overline{\mathbb{Q}}}\left(X ; U_{X}\right),
$$

où la dernière inégalité vient $\mathrm{du}$ fait qu'il existe un diviseur réalisant $\omega_{\overline{\mathbb{Q}}}\left(X ; U_{X}\right)$ défini sur $K$, d'après le corollaire 2.2 et le lemme 2.3 .

Le problème de Lehmer abélien consiste à montrer l'existence d'un réel $c>0$ tel que $m(K) \geq c\left[K: K \cap \mathbb{Q}^{\text {ab }}\right]^{-1}$. Dans cette direction, Amoroso et Zannier [AZ00] ont montré que dans le théorème de Dobrowolski on peut essentiellement remplacer le degré de $K$ sur $\mathbb{Q}$ par le degré $\left[K: K \cap \mathbb{Q}^{\text {ab }}\right]$. Récemment Amoroso et Delsinne [ADe06] on amélioré cette minoration en

$$
m(K) \geq \frac{1}{\left[K: K \cap \mathbb{Q}^{\mathrm{ab}}\right]} \frac{\log \log \left(5\left[K: K \cap \mathbb{Q}^{\mathrm{ab}}\right]\right)^{3}}{\log \left(2\left[K: K \cap \mathbb{Q}^{\mathrm{ab}}\right]\right)^{4}} .
$$


Suivant un argument dû à Amoroso et David, on peut montrer que la conjecture 1.1 entraîne une réponse positive au problème de Lehmer abélien. Nous généralisons cet argument pour montrer que la conjecture 1.1 est équivalente, à la constante $c(N)$ près, à son analogue sur la clôture abélienne de $\mathbb{Q}$ :

Proposition 2.5. Soit $X \subset \mathbb{G}_{m}^{N}$ une variété définie sur $\overline{\mathbb{Q}}$. Sous la conjecture 1.1 on a

$$
\widehat{\mu}^{\operatorname{ess}}(X) \geq c(N+1) \frac{\operatorname{deg}\left(U_{X}\right)}{\omega_{\mathbb{Q}^{\mathrm{ab}}}\left(X ; U_{X}\right)} .
$$

Réciproquement, la minoration (6) implique

$$
\widehat{\mu}^{\text {ess }}(X) \geq c(N+1) \frac{\operatorname{deg}\left(U_{X}^{\mathbb{Q}}\right)}{\omega_{\mathbb{Q}}\left(X ; U_{X}^{\mathbb{Q}}\right)} .
$$

Démonstration. Soit $K$ un corps de nombres sur lequel $X$ (et a fortiori $\left.U_{X}\right)$ sont définis, et soit $\xi \in \boldsymbol{\mu}^{\infty}$ telle que la variété de torsion $U_{X}$ soit définie sur l'extension cyclotomique $\mathbb{Q}(\xi)$. Soit $L$ le compositum de $K$ et $\mathbb{Q}(\xi)$; considérons $\widetilde{X}:=X \times\{\xi\} \subset \mathbb{G}_{m}^{N+1}$, qui est une variété définie sur $L$. Considérons la variété de torsion

$$
Y:=\bigcup_{\sigma \in \operatorname{Gal}(\mathbb{Q}(\xi) / \mathbb{Q})} \sigma\left(U_{X} \times\{\xi\}\right),
$$

qui, par construction, est définie sur $\mathbb{Q}$; on a $U_{\widetilde{X}}^{\mathbb{Q}} \subset Y$. En outre, il est clair que $U_{X} \times\{\xi\} \subset U_{\widetilde{X}}^{\mathbb{Q}}$, et en prenant la clôture galoisienne on obtient $Y=U_{\widetilde{X}}^{\mathbb{Q}}$. On a donc, par le lemme 2.4,

$$
\begin{aligned}
\operatorname{deg}\left(U_{\widetilde{X}}^{\mathbb{Q}}\right) & =[\mathbb{Q}(\xi): \mathbb{Q}] \operatorname{deg}\left(U_{X}\right), \\
\omega_{\mathbb{Q}}\left(\widetilde{X} ; U_{\widetilde{X}}^{\mathbb{Q}}\right) & =[\mathbb{Q}(\xi): \mathbb{Q}] \omega_{\mathbb{Q}(\xi)}\left(\widetilde{X} ; U_{X} \times\{\xi\}\right)=[\mathbb{Q}(\xi): \mathbb{Q}] \omega_{\mathbb{Q}(\xi)}\left(X ; U_{X}\right) .
\end{aligned}
$$

Et donc, en appliquant la conjecture 1.1 à $\widetilde{X}$, il vient

$$
\widehat{\mu}^{\operatorname{ess}}(X)=\widehat{\mu}^{\operatorname{ess}}(\widetilde{X}) \geq c(N+1) \frac{\operatorname{deg}\left(U_{\widetilde{X}}^{\mathbb{Q}}\right)}{\omega_{\mathbb{Q}}\left(\widetilde{X} ; U_{\widetilde{X}}^{\mathbb{Q}}\right)}=c(N+1) \frac{\operatorname{deg}\left(U_{X}\right)}{\omega_{\mathbb{Q}(\xi)}\left(X ; U_{X}\right)} .
$$

On conclut en prenant $\xi$ d'ordre suffisamment grand pour que $\omega_{\mathbb{Q}(\xi)}\left(X ; U_{X}\right)$ $=\omega_{\mathbb{Q}^{\mathrm{ab}}}\left(X ; U_{X}\right)$; c'est possible car $\mathbb{Q}^{\mathrm{ab}}=\mathbb{Q}\left(\boldsymbol{\mu}^{\infty}\right)$ (théorème de KroneckerWeber $)$.

Pour la réciproque on remarque que d'après le lemme 2.4 , si $K_{1} \subset \mathbb{Q}^{\text {ab }}$ désigne le corps de définition de $U_{X}$, on a

$$
\frac{\operatorname{deg}\left(U_{X}^{\mathbb{Q}}\right)}{\omega_{\mathbb{Q}}\left(X ; U_{X}^{\mathbb{Q}}\right)}=\frac{\operatorname{deg}\left(U_{X}\right)}{\omega_{K_{1}}\left(X ; U_{X}\right)} \leq \frac{\operatorname{deg}\left(U_{X}\right)}{\omega_{\mathbb{Q}^{\mathrm{ab}}}\left(X ; U_{X}\right)} . \cdot
$$


3. Degrés d'obstruction et réseaux. Dans ce paragraphe on étudie le degré d'obstruction d'un translaté de sous-tore dans un autre sous-tore, relativement à un corps algébriquement clos $\mathbb{k}$. Soit $\mathcal{B}=\left(b_{1}, \ldots, b_{N}\right) \in\left(\mathbb{Z}^{p}\right)^{N}$ tel que $L_{\mathcal{B}}=\mathbb{Z}^{p}$ et, comme précédemment, notons $T_{\mathcal{B}} \subset \mathbb{G}_{m}^{N}$ le sous-tore associé, image de l'application

$$
\varphi_{\mathcal{B}}: \mathbb{G}_{m}^{p} \rightarrow \mathbb{G}_{m}^{N}, \quad s \mapsto\left(s^{b_{1}}, \ldots, s^{b_{N}}\right),
$$

et $\beta T_{\mathcal{B}}$ son translaté par un point $\beta \in \mathbb{G}_{m}^{N}$. Reprenons l'application $M_{\mathcal{B}}$ introduite au début du $\S 2.1$ (formule (4)) ; le noyau $\operatorname{ker}\left(M_{\mathcal{B}}\right) \subset \mathbb{Z}^{N}$ est un sous-module de rang $N-n$, saturé, c'est-à-dire tel que le quotient $\mathbb{Z}^{N} / \operatorname{ker}\left(M_{\mathcal{B}}\right)$ soit sans torsion. Les idéaux de définition de $T_{\mathcal{B}}$ et de $\beta T_{\mathcal{B}}$ dans $\mathbb{k}\left[x_{1}^{ \pm 1}, \ldots, x_{N}^{ \pm 1}\right]$ s'écrivent $[\mathrm{ES} 96]$

$$
I\left(T_{\mathcal{B}}\right)=\left(x^{\lambda}-1: \lambda \in \operatorname{ker}\left(M_{\mathcal{B}}\right)\right), \quad I\left(\beta T_{\mathcal{B}}\right)=\left(x^{\lambda}-\beta^{\lambda}: \lambda \in \operatorname{ker}\left(M_{\mathcal{B}}\right)\right) .
$$

Ainsi, le sous-tore $T_{\mathcal{B}}$ ne dépend que de $\operatorname{ker}\left(M_{\mathcal{B}}\right)$. Plus généralement, le translaté $\beta T_{\mathcal{B}}$ ne dépend que de $\operatorname{ker}\left(M_{\mathcal{B}}\right)$ et de l'homomorphisme $\operatorname{ker}\left(M_{\mathcal{B}}\right) \rightarrow \mathbb{k}^{\times}$, $\lambda \mapsto \beta^{\lambda}$.

Réciproquement, soit $\Gamma \subset \mathbb{Z}^{N}$ un sous-module saturé muni d'un caractère $\varrho$, c'est-à-dire un homomorphisme $\varrho: \Gamma \rightarrow \mathbb{k}^{\times}$. Ces données définissent un idéal binomial

$$
I(\Gamma, \varrho):=\left(x^{\lambda}-\varrho(\lambda): \lambda \in \Gamma\right) \subset \mathbb{k}\left[x_{1}^{ \pm 1}, \ldots, x_{N}^{ \pm 1}\right] .
$$

On peut vérifier que tout sous-module saturé de $\mathbb{Z}^{N}$ se réalise comme $\operatorname{ker}\left(M_{\mathcal{B}}\right)$ pour un certain $\mathcal{B} \in\left(\mathbb{Z}^{p}\right)^{N}$ du type envisagé ci-dessus, et que tout caractère d'un tel module se réalise par un point $\beta \in \mathbb{G}_{m}^{N}$ (on peut même choisir $\left.\beta \in \varrho(\Gamma)^{N}\right)$. Les correspondances

$$
(\Gamma, \varrho) \mapsto I(\Gamma, \varrho) \quad \text { et } \quad I \mapsto Z(I)
$$

sont des bijections entre les sous-modules saturés $\Gamma$ de $\mathbb{Z}^{N}$ munis d'un caractère $\varrho$, les idéaux premiers binomiaux de $\mathbb{k}\left[x_{1}^{ \pm 1}, \ldots, x_{N}^{ \pm 1}\right]$ ne contenant aucune des variables $x_{j}$, et les translatés de sous-tores de $\mathbb{G}_{m}^{N}$ [ES96, Cor. 2.6].

On écrira aussi $T(\Gamma, \varrho)$ pour le translaté de sous-tore associé au couple $(\Gamma, \varrho)$. Le caractère trivial $\varrho=1$ correspond à un sous-tore, que l'on note alors simplement $T(\Gamma)$, et ceux pour lesquels $\varrho(\Gamma) \subset \boldsymbol{\mu}^{\infty}$ aux variétés de torsion. Réciproquement, pour un translaté de sous-tore $X \subset \mathbb{G}_{m}^{N}$ on désigne par $\Gamma_{X}$ et $\varrho_{X}$ le sous-module et le caractère associés. La représentation par sousmodules saturés et caractères correspond donc aux équations des translatés de sous-tores. Dans ce paragraphe on privilégiera cette représentation, sauf dans la proposition 3.6.

Le translaté $T(\Gamma, \varrho)$ est défini sur un sous-corps $K$ si et seulement si $\varrho(\Gamma) \subset K^{\times}$. Ceci équivaut à ce qu'il existe $\beta \in\left(K^{\times}\right)^{N}$ tel que $\varrho(\lambda)=\beta^{\lambda}$ pour tout $\lambda \in \Gamma$, c'est-à-dire à ce que $T(\Gamma, \varrho)$ contienne un point $K$-rationnel. En effet, comme $\Gamma$ est saturé l'homomorphisme $\varrho$ s'étend en $\varrho: \mathbb{Z}^{N} \rightarrow K^{\times}$, 
posant alors $\beta_{1}, \ldots, \beta_{N} \in K^{\times}$les images par $\varrho$ des éléments de la base canonique de $\mathbb{Z}^{N}$ on vérifie que $\varrho$ s'écrit bien $\lambda \mapsto \beta^{\lambda}$.

Soient $X, Y \subset \mathbb{G}_{m}^{N}$ des translatés de sous-tores. La condition $X \subset Y$ équivaut à

$$
\Gamma_{X} \supset \Gamma_{Y} \quad \text { et }\left.\quad\left(\varrho_{X}\right)\right|_{\Gamma_{Y}}=\varrho_{Y} .
$$

En particulier, $X \subset Y$ entraîne $T\left(\Gamma_{X}\right) \subset T\left(\Gamma_{Y}\right)$. De plus, $Y$ est la variété de torsion minimale contenant $X$ si et seulement si $\varrho_{Y}\left(\Gamma_{Y}\right) \subset \boldsymbol{\mu}^{\infty}$ et $\varrho_{X}(\lambda) \notin$ $\boldsymbol{\mu}^{\infty}$ pour tout $\lambda \in \Gamma_{X} \backslash \Gamma_{Y}$. Autrement dit, cette variété de torsion minimale est caractérisée par

$$
\Gamma_{Y}=\left\{\lambda \in \Gamma_{X}: \varrho_{X}(\lambda) \in \boldsymbol{\mu}^{\infty}\right\} \quad \text { et } \quad \varrho_{Y}=\left.\left(\varrho_{X}\right)\right|_{\Gamma_{Y}} .
$$

Le degré d'obstruction des translatés de sous-tores dans des sous-tores relativement à $\mathbb{k}$ se réalise par un diviseur qui lui aussi est un translaté de sous-tore :

Proposition 3.1. Soient $X, Y \subset \mathbb{G}_{m}^{N}$ des translatés de sous-tores tels que $X \subsetneq Y$. Alors il existe $\lambda \in \Gamma_{X} \backslash \Gamma_{Y}$ tel que

$$
\omega_{\mathbb{k}}(X ; Y)=\operatorname{deg}\left(\operatorname{div}\left(x^{\lambda}-\varrho_{X}(\lambda)\right) \cdot Y\right) .
$$

Démonstration. Soient $I(X), I(Y) \subset \mathbb{k}\left[x_{1}^{ \pm 1}, \ldots, x_{N}^{ \pm 1}\right]$ les idéaux de définition de $X$ et de $Y$ respectivement. L'anneau $\mathbb{k}[Y]$ est factoriel car isomorphe à un anneau du type $\mathbb{k}\left[y_{1}^{ \pm 1}, \ldots, y_{p}^{ \pm 1}\right]$, et donc tout diviseur est principal. Soit donc $F \in I(X) \backslash I(Y)$ réalisant $\omega_{\mathbb{k}}(X ; Y)$; on peut écrire $F$ modulo $I(Y)$ sous la forme

$$
F=\sum_{\nu \in \mathbb{Z}^{N} / \Gamma_{Y}} f_{\nu} x^{\nu}=\sum_{\sigma \in \mathbb{Z}^{N} / \Gamma_{X}} x^{\sigma} F_{\sigma}
$$

avec $F_{\sigma}=\sum_{\tau \in \Gamma_{X} / \Gamma_{Y}} f_{\sigma+\tau} x^{\tau}$ pour $\sigma \in \mathbb{Z}^{N} / \Gamma_{X}$. Les monômes $x^{\sigma}(\sigma \in$ $\left.\mathbb{Z}^{N} / \Gamma_{X}\right)$ sont linéairement indépendants modulo $I(X)$ et donc, comme $F \in$ $I(X)$, on a $F_{\sigma} \in I(X)$ pour tout $\sigma$, de plus il existe $\sigma_{0} \in \mathbb{Z}^{N} / \Gamma_{X}$ tel que $F_{\sigma_{0}} \neq 0$. Ce polynôme ne peut pas être réduit à un monôme car $I(X)$ ne contient pas de monômes et il existe donc $a, b \in \Gamma_{X} / \Gamma_{Y}, a \neq b$, tels que

$$
\mathrm{N}(F) \supset \overline{a b}
$$

où $\overline{a b} \subset \mathbb{R}^{N}$ désigne le segment d'extrémités $a, b$. Soit $\lambda:=a-b$; on déduit de la proposition 2.1 et de la monotonie du volume mixte par rapport à l'inclusion [Ewa96, Thm. 4.12] que le degré $\operatorname{de} \operatorname{div}\left(F_{\sigma_{0}}\right) \cdot Y$ est supérieur ou égal au degré du diviseur $\operatorname{div}\left(x^{\lambda}-\varrho_{X}(\lambda)\right) \cdot Y$, et donc que $\omega_{\mathbb{k}}(X ; Y)$ est réalisé par un diviseur qui est un translaté de sous-tore de la forme indiquée.

On notera que pour un sous-corps $K$ qui n'est pas algébriquement clos, le degré d'obstruction d'un translaté de sous-tore dans un sous-tore n'est pas nécessairement réalisé par un diviseur qui est un translaté de sous-tore. Voici un exemple, aimablement fourni par David : 
Exemple 3.2. Soit $\xi \in \overline{\mathbb{Q}} \backslash \mathbb{Q}$ et posons

$$
X:=(\xi, 1-\xi) \in \mathbb{G}_{m}^{2}, \quad Y:=\mathbb{G}_{m}^{2} .
$$

On a $\omega_{\mathbb{Q}}(X ; Y)=1$ réalisé par la droite d'équation $x+y=1$, et on vérifie qu'il n'y a pas d'équation binomiale de degré 1 , définie sur $\mathbb{Q}$ et contenant $X$. Par ailleurs, on a $\omega_{\overline{\mathbb{Q}}}(X ; Y)=1$, qui est bien sûr réalisé par la droite d'équation $x+y=1$, mais aussi par des diviseurs translatés de sous-tores, d'équations $x-\xi=0, y-(1-\xi)=0$ ou encore $(1-\xi) x-\xi y=0$.

De plus, on vérifie $U_{X}^{\mathbb{Q}}=U_{X}=Y=\mathbb{G}_{m}^{2}$ et la minoration de la conjecture 1.1 s'écrit $\widehat{\mu}^{\operatorname{ess}}(X) \geq c(2)$, tandis que celle du théorème $1.3, \widehat{\mu}^{\text {ess }}(X) \geq$ $m(\mathbb{Q}(\xi)) / 8 \sqrt{2}$, donne un minorant pouvant être rendu arbitrairement petit (en variant $\xi$ ). Ceci montre que le théorème 1.3 ne peut entrainer la conjecture 1.1 .

Soient $X \subset Y$ des translatés de sous-tores de dimension $n$ et $p$ respectivement et posons

$$
\Gamma_{X, Y}:=\Gamma_{X} / \Gamma_{Y}
$$

le module quotient, qui est un $\mathbb{Z}$-module libre de rang $(N-n)-(N-p)=$ $p-n$. Considérons aussi l'espace linéaire $\Gamma_{X, Y}^{\mathbb{R}}:=\Gamma_{X, Y} \otimes_{\mathbb{Z}} \mathbb{R}$ muni de la métrique quotient $\|\cdot\|_{\perp}$ induite de la métrique euclidienne $\|\cdot\|_{2}$ par identification avec l'orthogonal de $\Gamma_{Y}^{\mathbb{R}}$ dans $\Gamma_{X}^{\mathbb{R}}$. En particulier, $\Gamma_{X, Y}$ est un réseau de $\Gamma_{X, Y}^{\mathbb{R}}$.

Proposition 3.3. Soient $X \subsetneq Y$ des translatés de sous-tores de dimension $n$ et $p$ respectivement. Alors pour tout $\lambda \in \Gamma_{X} \backslash \Gamma_{Y}$ on a

$$
\left(\left(\begin{array}{c}
N \\
p
\end{array}\right)\left(\begin{array}{c}
N \\
p-1
\end{array}\right)\right)^{-1 / 2} \leq \frac{\operatorname{deg}\left(\operatorname{div}\left(x^{\lambda}-\varrho_{X}(\lambda)\right) \cdot Y\right)}{\|\lambda\|_{\perp} \operatorname{deg}(Y)} \leq\left(\left(\begin{array}{c}
N \\
p
\end{array}\right)\left(\begin{array}{c}
N \\
p-1
\end{array}\right)\right)^{1 / 2} .
$$

Démonstration. Posons $W:=\operatorname{div}\left(x^{\lambda}-\varrho_{X}(\lambda)\right) \cdot Y$. Quitte à diviser $\lambda$ par un entier, ce qui divise également $\operatorname{deg}(W)$ et $\|\lambda\|_{\perp}$ par ce même entier, on peut supposer sans perte de généralité que $\Gamma_{W}:=\Gamma_{Y}+\mathbb{Z} \lambda$ est un $\mathbb{Z}$-module saturé et donc [PS05, §3]

$$
\begin{aligned}
\left(\begin{array}{c}
N \\
p-1
\end{array}\right)^{-1 / 2} \operatorname{Vol}_{N-p+1}\left(\Gamma_{W}^{\mathbb{R}} / \Gamma_{W}\right) & \leq \operatorname{deg}(W) \\
& \leq\left(\begin{array}{c}
N \\
p-1
\end{array}\right)^{1 / 2} \operatorname{Vol}_{N-p+1}\left(\Gamma_{W}^{\mathbb{R}} / \Gamma_{W}\right) .
\end{aligned}
$$

De même

$$
\left(\begin{array}{c}
N \\
p
\end{array}\right)^{-1 / 2} \operatorname{Vol}_{N-p}\left(\Gamma_{Y}^{\mathbb{R}} / \Gamma_{Y}\right) \leq \operatorname{deg}(Y) \leq\left(\begin{array}{c}
N \\
p
\end{array}\right)^{1 / 2} \operatorname{Vol}_{N-p}\left(\Gamma_{Y}^{\mathbb{R}} / \Gamma_{Y}\right) .
$$

Mais $\operatorname{Vol}_{N-p+1}\left(\Gamma_{W}^{\mathbb{R}} / \Gamma_{W}\right)=\|\lambda\|_{\perp} \operatorname{Vol}_{N-p}\left(\Gamma_{Y}^{\mathbb{R}} / \Gamma_{Y}\right)$ et les inégalités cherchées en résultent. 
Lemme 3.4. Avec les notations de la proposition 3.3, le premier minimum du réseau $\Gamma_{X, Y}$ est majoré par

$$
\frac{2}{\sqrt{\pi}}\left(\left(\begin{array}{c}
N \\
n
\end{array}\right)\left(\begin{array}{c}
N \\
p
\end{array}\right) \boldsymbol{\Gamma}\left(1+\frac{p-n}{2}\right)^{2}\right)^{1 / 2(p-n)}\left(\frac{\operatorname{deg}(X)}{\operatorname{deg}(Y)}\right)^{1 /(p-n)},
$$

où $\boldsymbol{\Gamma}$ désigne la fonction gamma d'Euler.

Démonstration. Le théorème de Minkowski [Cas71, §VIII.4.3, Thm.V] entraîne que le premier minimum de la norme $\|\cdot\|_{\perp}$ sur le réseau $\Gamma_{X, Y}$ est majoré par

$$
\frac{2}{\sqrt{\pi}} \boldsymbol{\Gamma}\left(1+\frac{p-n}{2}\right)^{1 /(p-n)} \operatorname{Vol}_{p-n}\left(\Gamma_{X, Y}^{\mathbb{R}} / \Gamma_{X, Y}\right)^{1 /(p-n)} .
$$

Mais d'après [PS05, §3] (voir aussi inégalité (8) ci-dessus),

$$
\operatorname{Vol}_{p-n}\left(\Gamma_{X, Y}^{\mathbb{R}} / \Gamma_{X, Y}\right)=\frac{\operatorname{Vol}_{N-n}\left(\Gamma_{X}^{\mathbb{R}} / \Gamma_{X}\right)}{\operatorname{Vol}_{N-p}\left(\Gamma_{Y}^{\mathbb{R}} / \Gamma_{Y}\right)} \leq\left(\left(\begin{array}{c}
N \\
n
\end{array}\right)\left(\begin{array}{c}
N \\
p
\end{array}\right)\right)^{1 / 2} \frac{\operatorname{deg}(X)}{\operatorname{deg}(Y)},
$$

d'où l'énoncé.

En combinant le lemme 3.4 avec l'inégalité de droite de la proposition 3.3 appliquée au premier minimum du réseau $\Gamma_{X, Y}$ on trouve :

Corollaire 3.5. Soient $X \subsetneq Y$ des translatés de sous-tores de dimension $n$ et $p$ respectivement. Alors

$$
\omega_{\mathbb{k}}(X ; Y) \leq c_{3}(N, p, n) \operatorname{deg}(X)^{1 /(p-n)} \operatorname{deg}(Y)^{1-1 /(p-n)}
$$

avec

$$
\begin{aligned}
c_{3}(N, p, n) & :=\frac{2}{\sqrt{\pi}}\left(\left(\begin{array}{c}
N \\
p
\end{array}\right)\left(\begin{array}{c}
N \\
p-1
\end{array}\right)\right)^{1 / 2}\left(\left(\begin{array}{c}
N \\
n
\end{array}\right)\left(\begin{array}{c}
N \\
p
\end{array}\right) \boldsymbol{\Gamma}\left(1+\frac{p-n}{2}\right)^{2}\right)^{1 / 2(p-n)} \\
& \leq 4^{N} N^{3 / 2}
\end{aligned}
$$

En particulier, lorsque $X$ est réduit à un point $\alpha \in \mathbb{G}_{m}^{N}$ on a $\omega_{\mathbb{k}}(\alpha ; Y) \leq$ $\left(2^{N} N\right)^{3 / 2} \operatorname{deg}(Y)^{1-1 / p}$.

Il est naturel de se demander si cette majoration s'étend à d'autres variétés, c'est-à-dire si

$$
\omega_{\mathbb{k}}(X ; Y) \leq c(N) \operatorname{deg}(X)^{1 /(p-n)} \operatorname{deg}(Y)^{1-1 /(p-n)}
$$

pour des variétés projectives $X \subsetneq Y$ de dimension $n$ et $p$ respectivement. Chardin [Cha89] a démontré un tel résultat pour $Y=\mathbb{P}^{N}$ et $X \subset \mathbb{P}^{N}$ quelconque. Cependant, il semblerait qu'une généralisation ne soit possible qu'en imposant des conditions sur la régularité de Castelnuovo-Mumford de $Y$.

Il serait également intéressant de remplacer les estimations de la proposition 3.3 par une égalité. Est-ce possible en modifiant convenablement la métrique de $\Gamma_{X, Y}$ ? Le résultat suivant fournit une expression alternative 
exacte pour le degré d'obstruction, cependant elle ne nous est pas utile pour la suite.

Proposition 3.6. Soit $Y \subset \mathbb{G}_{m}^{N}$ un translaté de sous-tore, $\mathcal{B} \in\left(\mathbb{Z}^{p}\right)^{N}$ satisfaisant $L_{\mathcal{B}}=\mathbb{Z}^{p}$ et $\beta \in \mathbb{G}_{m}^{N}$, tels que $Y=\beta T_{\mathcal{B}}$. Soit $\lambda \in \mathbb{Z}^{N} \backslash \Gamma_{Y}$ tel que $\lambda \mathbb{Z}+\Gamma_{Y}$ soit saturé et $\gamma \in \mathbb{R}^{\times}$quelconque. Alors

$$
\operatorname{deg}\left(\operatorname{div}\left(x^{\lambda}-\gamma\right) \cdot Y\right)=(p-1) ! \operatorname{Vol}_{p-1}\left(\pi_{\lambda}\left(Q_{\mathcal{B}}\right)\right)\left\|M_{\mathcal{B}}(\lambda)\right\|_{2},
$$

où $Q_{\mathcal{B}} \subset \mathbb{R}^{p}$ désigne l'enveloppe convexe des points $\mathbf{0}, b_{1}, \ldots, b_{N}$, $\pi_{\lambda}$ la projection orthogonale $\pi_{\lambda}: \mathbb{R}^{p} \rightarrow M_{\mathcal{B}}(\lambda)^{\perp} \cong \mathbb{R}^{p-1}$ et $\|\cdot\|_{2}$ la norme euclidienne.

Démonstration. Posons $W:=\operatorname{div}\left(x^{\lambda}-\gamma\right) \cdot Y$. Comme $\lambda \mathbb{Z}+\Gamma_{Y}$ est saturé, $W$ est réduit et irréductible de dimension $p-1$; c'est donc un translaté de sous-tore.

Soit $E:=M_{\mathcal{B}}(\lambda)^{\perp}$ et $E^{\mathbb{Z}}:=\pi_{\lambda}\left(\mathbb{Z}^{p}\right)$; alors $E^{\mathbb{Z}}$ est un réseau de $E$ et on vérifie $\operatorname{Vol}_{p-1}\left(E / E^{\mathbb{Z}}\right)=\left\|M_{\mathcal{B}}(\lambda)\right\|_{2}^{-1}$. Posons maintenant $c_{j}:=\pi_{\lambda}\left(b_{j}\right)$ pour $j=1, \ldots, N$ et $\mathcal{C}:=\left(c_{1}, \ldots, c_{N}\right)$, de sorte que $L_{\mathcal{C}}=E^{\mathbb{Z}}$, et soit $\eta \in \mathbb{G}_{m}^{N}$ tel que $\gamma=\eta^{\lambda}$, de sorte que $\eta T_{\mathcal{C}}$ est de dimension $p-1$. Le translaté de sous-tore

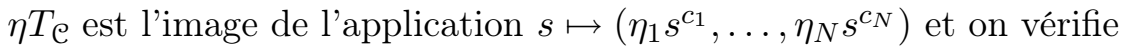

$$
\left(\eta_{1} s^{c_{1}}, \ldots, \eta_{N} s^{c_{N}}\right)^{\lambda}-\eta^{\lambda}=\eta^{\lambda}\left(s^{c_{1} \lambda_{1}+\cdots+c_{N} \lambda_{N}}-1\right) .
$$

Mais $c_{1} \lambda_{1}+\cdots+c_{N} \lambda_{N}=\pi_{\lambda}\left(b_{1}\right) \lambda_{1}+\cdots+\pi_{\lambda}\left(b_{N}\right) \lambda_{N}=\pi_{\lambda}\left(M_{\mathcal{B}}(\lambda)\right)=0$ d'où $\eta T_{\mathcal{C}} \subset W$ et donc $\eta T_{\mathcal{C}}=W$. Le calcul de degré découle alors du calcul du volume du polytope $P:=\operatorname{Conv}\left(\mathbf{0}, c_{1}, \ldots, c_{N}\right)=\pi_{\lambda}\left(Q_{\mathcal{B}}\right)$ :

$$
\operatorname{deg}(W)=(p-1) ! \frac{\operatorname{Vol}_{p-1}(P)}{\operatorname{Vol}_{p-1}\left(E / E^{\mathbb{Z}}\right)}=(p-1) ! \operatorname{Vol}_{p-1}\left(\pi_{\lambda}\left(Q_{\mathcal{B}}\right)\right)\left\|M_{\mathcal{B}}(\lambda)\right\|_{2} .
$$

Remarque 3.7. En combinant les propositions 3.6 et 2.1 on vérifie

$$
\operatorname{MV}(\left[0, M_{\mathcal{B}}(\lambda)\right], \underbrace{Q_{\mathcal{B}}, \ldots, Q_{\mathcal{B}}}_{p-1 \text { fois }})=(p-1) ! \operatorname{Vol}_{p-1}\left(\pi_{\lambda}\left(Q_{\mathcal{B}}\right)\right)\left\|M_{\mathcal{B}}(\lambda)\right\|_{2} .
$$

Cette identité se déduit également de la formule générale suivante pour un convexe $Q$ quelconque en lieu et place du segment $\left[0, M_{\mathcal{B}}(\lambda)\right]$ :

$$
\operatorname{MV}(Q, \underbrace{Q_{\mathcal{B}}, \ldots, Q_{\mathcal{B}}}_{p-1 \text { fois }})=(p-1) ! \sum_{w \in S^{p-1}} a_{w}(Q) \operatorname{Vol}_{p-1}\left(Q_{w}\right),
$$

où $w$ parcourt la sphère unité de $\mathbb{R}^{p}, a_{w}(Q):=\max \{\langle w, q\rangle: q \in Q\}$ et $Q_{w}:=\left\{q \in Q_{\mathcal{B}}:\langle w, q\rangle=a_{w}(q)\right\}$ (voir [Ewa96, Ch. IV, Thm. 4.10]).

\section{Démonstrations du théorème 1.3 et des corollaires 1.4 et 1.5 .} Nous donnons ici les preuves des résultats énoncés dans l'introduction. Remarquons tout de même que dans le cas des hypersurfaces $(n=N-1)$ le théorème 1.3 est trivial : dans ce cas un translaté de sous-tore qui n'est pas de torsion est de la forme $X=Z\left(x^{b}-\lambda\right)$ pour un vecteur $b \in \mathbb{Z}^{N}$ à 
coordonnées premières entre elles et $\lambda \in K^{\times} \backslash \boldsymbol{\mu}^{\infty}$, et on a $U_{X}=\mathbb{G}_{m}^{N}$. Donc, d'après [PS05, prop. VI.4],

$$
\widehat{\mu}^{\mathrm{ess}}(X)=\frac{\widehat{h}(X)}{\operatorname{deg}(X)}=\frac{\widehat{h}(\lambda)}{\omega_{\overline{\mathbb{Q}}}\left(X ; \mathbb{G}_{m}^{N}\right)} \geq m(K) \frac{\operatorname{deg}\left(U_{X}\right)}{\omega_{\overline{\mathbb{Q}}}\left(X ; U_{X}\right)},
$$

où $\widehat{h}(X)$ désigne la hauteur normalisée de l'hypersurface $X$.

Démonstration du théorème 1.3. On écrit $X=\alpha T$ et $U=U_{X}=\xi T^{\prime}$ pour certains sous-tores $T, T^{\prime}, \alpha \in\left(K^{\times}\right)^{N}$ et $\xi \in\left(\boldsymbol{\mu}^{\infty}\right)^{N}$. On pose $n=$ $\operatorname{dim}(X), p=\operatorname{dim}\left(U_{X}\right)$ et on suppose sans perte de généralité $n<p$.

Soit $\lambda \in \Gamma_{X} \backslash \Gamma_{U}$. Écrivons $\lambda=c+b$ avec $b \in \Gamma_{U}^{\mathbb{Q}}$ et $c \in\left(\Gamma_{U}^{\mathbb{Q}}\right)^{\perp}$, où $\Gamma^{\mathbb{Q}}:=\Gamma \otimes_{\mathbb{Z}} \mathbb{Q}$. Soit encore $\zeta=\left(\zeta_{1}, \ldots, \zeta_{N}\right) \in X$ un point quelconque. Alors $\zeta=\alpha \theta$ pour un certain $\theta \in T$, d'où, puisque $\theta^{\lambda}=1$,

$$
\zeta^{\lambda}=(\alpha \theta)^{\lambda}=\alpha^{\lambda} \quad \text { pour tout } \lambda \in \Gamma_{X} .
$$

De plus $\zeta^{b} \in \boldsymbol{\mu}^{\infty}$ car $\zeta \in \xi T^{\prime}, b \in \Gamma_{U}^{\mathbb{Q}}$ et pour tout entier $m$ tel que $m b \in \Gamma_{U}$ on a $\left(\zeta^{b}\right)^{m}=\xi^{m b}$. Comme $\lambda \notin \Gamma_{U}$ il vient que $\zeta^{\lambda}$ (et par la suite $\zeta^{c}$ ) n'est pas une racine de l'unité, à cause de la minimalité de $U$ parmi les variétés de torsion contenant $X$ (voir à ce propos la discussion avant la proposition 3.1).

Soit $L \supset K$ un corps de nombres contenant $\zeta_{1}^{c_{1}}, \ldots, \zeta_{N}^{c_{N}}$. Comme $\alpha^{\lambda}$ et $\zeta^{c}$ ne diffèrent que du facteur $\zeta^{b}$ qui est une racine de l'unité, pour toute place $v \in M_{L}$ on a

$$
\begin{aligned}
\left.|\log | \alpha^{\lambda}\right|_{v} \mid & =\left.|\log | \zeta^{c}\right|_{v}|=| c_{1} \log \left|\zeta_{1}\right|_{v}+\cdots+c_{N} \log \left|\zeta_{N}\right|_{v} \mid \\
& \leq\left(\sum_{i=1}^{N}\left|c_{i}\right|\right) \max \left(\left.|\log | \zeta_{1}\right|_{v}|, \ldots,| \log \left|\zeta_{N}\right|_{v} \mid\right) \\
& \leq \sqrt{p-n}\|\lambda\|_{\perp} \max \left(\left.|\log | \zeta_{1}\right|_{v}|, \ldots,| \log \left|\zeta_{N}\right|_{v} \mid\right)
\end{aligned}
$$

car $c$ est la projection de $\lambda$ sur l'orthogonal $\left(\Gamma_{U}^{\mathbb{Q}}\right)^{\perp}$ de $\Gamma_{U}^{\mathbb{Q}} \operatorname{dans} \Gamma_{X}^{\mathbb{Q}}$ et $\operatorname{dim}_{\mathbb{Q}}\left(\Gamma_{U}^{\mathbb{Q}}\right)^{\perp}=p-n$. D'où, en sommant sur toutes les places de $L$,

$$
2 \widehat{h}\left(\alpha^{\lambda}\right)=\left.\sum_{v \in M_{L}} \frac{\left[L_{v}: \mathbb{Q}_{v}\right]}{[L: \mathbb{Q}]}|\log | \alpha^{\lambda}\right|_{v} \mid \leq \sqrt{p-n}\|\lambda\|_{\perp}\left(\widehat{h}(\zeta)+\widehat{h}\left(\zeta^{-1}\right)\right) .
$$

Et comme

$$
\begin{aligned}
\widehat{h}\left(\zeta^{-1}\right) & =\sum_{v} \frac{\left[L_{v}: \mathbb{Q} v\right]}{[L: \mathbb{Q}]} \max \left\{0,-\log \left|\zeta_{1}\right|_{v}, \ldots,-\log \left|\zeta_{N}\right|_{v}\right\} \\
& \leq \widehat{h}\left(\zeta_{1}^{-1}\right)+\cdots+\widehat{h}\left(\zeta_{N}^{-1}\right) \leq N \widehat{h}(\zeta)
\end{aligned}
$$

on a

$$
\widehat{h}(\zeta) \geq \frac{2}{(N+1) \sqrt{p-n}} \frac{\widehat{h}\left(\alpha^{\lambda}\right)}{\|\lambda\|_{\perp}} \geq \frac{2}{(N+1) \sqrt{p-n}} \frac{m(K)}{\|\lambda\|_{\perp}}
$$


par définition de $m(K)$, car $\alpha^{\lambda} \in K^{\times} \backslash \boldsymbol{\mu}^{\infty}$. La proposition 3.1 assure l'existence de $\lambda \in \Gamma_{X} \backslash \Gamma_{U}$ réalisant le degré d'obstruction $\omega_{\overline{\mathbb{Q}}}(X ; U)$ et, par la proposition 3.3,

$$
\|\lambda\|_{\perp} \leq\left(\left(\begin{array}{c}
N \\
p
\end{array}\right)\left(\begin{array}{c}
N \\
p-1
\end{array}\right)\right)^{1 / 2} \frac{\omega_{\overline{\mathbb{Q}}}(X ; U)}{\operatorname{deg}(U)},
$$

d'où finalement

$$
\widehat{h}(\zeta) \geq c(N, p, n) m(K) \frac{\operatorname{deg}(U)}{\omega_{\overline{\mathbb{Q}}}(X ; U)}
$$

avec $c(N, p, n)=2\left((N+1)^{2}(p-n)\left(\begin{array}{c}N \\ p\end{array}\right)\left(\begin{array}{c}N \\ p-1\end{array}\right)\right)^{-1 / 2} \geq N^{-3 / 2} 2^{-N}$, ce qui achève la démonstration du théorème 1.3.

Remarque 4.1. En minorant $m(K)$ par $c[K: \mathbb{Q}]^{-1}$ selon la réponse attendue à la question de Lehmer, on est tenté de considérer le degré d'obstruction modifié suivant, pour $X$ et $U$ comme dans la démonstration ci-dessus :

$$
\varpi(X ; U):=\inf \left\{\left[\mathbb{Q}\left(\alpha^{\lambda}\right): \mathbb{Q}\right] \operatorname{deg}\left(\operatorname{div}\left(x^{\lambda}-\alpha^{\lambda}\right) \cdot U\right): \lambda \in \Gamma_{X} \backslash \Gamma_{U}\right\} .
$$

La démonstration ci-dessus entraîne alors

$$
\widehat{\mu}^{\mathrm{abs}}(X) \geq c \cdot c_{1}(N) \cdot \frac{\operatorname{deg}\left(U_{X}\right)}{\varpi\left(X ; U_{X}\right)} .
$$

Lemme 4.2. Soit $X$ un translaté de sous-tore défini sur un corps $C M$. Alors la plus petite variété de torsion $G_{X}$ contenant $X \cdot \bar{X}$ est un sous-tore.

Démonstration. Soit $K$ le corps de définition de $X$. Écrivons $X=\alpha T$ et $G_{X}=\xi T^{\prime}$ pour certains sous-tores $T, T^{\prime}, \alpha \in\left(K^{\times}\right)^{N}$ et $\xi \in\left(\boldsymbol{\mu}^{\infty}\right)^{N}$. On a $X \cdot \bar{X}=|\alpha|^{2} T$, donc pour tout $\lambda \in \Gamma_{T^{\prime}}$ on a $|\alpha|^{2 \lambda}=\xi^{\lambda} \in \mathbb{R}_{+} \cap \boldsymbol{\mu}^{\infty}$, d'où $\xi^{\lambda}=1$; autrement dit, $\xi \in T^{\prime}$ et donc $G_{X}=\xi T^{\prime}=T^{\prime}$ est un sous-tore.

Démonstration du corollaire 1.4. Lorsque $K$ est un corps totalement réel ou une extension abélienne de $\mathbb{Q}$ on a $m(K) \geq \frac{1}{2} \log ((1+\sqrt{5}) / 2)$ d'après $(2)$ ou $m(K) \geq \log (5) / 12$ d'après (3), et on reporte directement dans le théorème 1.3 .

Si $K$ est un corps CM, la plus petite sous-variété de torsion contenant $Y:=X \cdot \bar{X}$ est un sous-tore $G_{X}$, d'après le lemme 4.2. De plus, $Y$ est définie sur le sous-corps totalement réel $K_{0}$ de $K$. Mais on a $m\left(K_{0}\right) \geq \frac{1}{2} \log \left(\frac{1+\sqrt{5}}{2}\right)$ d'après (2) et, si $Y$ n'est pas une variété de torsion, on déduit du théorème 1.3 que

$$
\widehat{\mu}^{\mathrm{ess}}(Y) \geq \frac{c_{1}(N)}{2} \log \left(\frac{1+\sqrt{5}}{2}\right) \frac{\operatorname{deg}\left(G_{X}\right)}{\omega_{\overline{\mathbb{Q}}}\left(Y ; G_{X}\right)} .
$$

Maintenant, si $Y$ est une variété de torsion on a $Y=G_{X}$ et $\omega_{\overline{\mathbb{Q}}}\left(Y ; G_{X}\right)$ $=\infty$, la minoration ci-dessus est donc encore vraie dans ce cas. Finalement, on remarque $\widehat{\mu}^{\text {ess }}(Y) \leq 2 \widehat{\mu}^{\text {ess }}(X)$ pour conclure la démonstration. 
On pourrait directement déduire le corollaire 1.5 du théorème 1.3, mais nous préférons reprendre en partie la preuve afin d'obtenir une meilleure constante numérique.

Démonstration du corollaire 1.5. On combine (9) dans la preuve du théorème 1.3 ci-dessus et le lemme 3.4 en choisissant $\lambda$ réalisant le premier minimum de $\Gamma_{X} / \Gamma_{U}$, ce qui donne $\left(X=\alpha T, U_{X}=\xi T^{\prime}\right)$

$$
\begin{aligned}
\widehat{\mu}^{\text {abs }}(\alpha T) \geq & \frac{2}{(N+1) \sqrt{p-n}} m(K) \frac{1}{\|\lambda\|_{\perp}} \\
\geq & \frac{\sqrt{\pi}}{(N+1) \sqrt{p-n}}\left(\left(\begin{array}{c}
N \\
n
\end{array}\right)\left(\begin{array}{c}
N \\
p
\end{array}\right) \Gamma\left(1+\frac{p-n}{2}\right)^{2}\right)^{-1 / 2(p-n)} \\
& \times m(K)\left(\frac{\operatorname{deg}\left(T^{\prime}\right)}{\operatorname{deg}(T)}\right)^{1 /(p-n)} \\
\geq & c_{4}(N) m(K)\left(\frac{\operatorname{deg}\left(U_{X}\right)}{\operatorname{deg}(X)}\right)^{1 /(p-n)},
\end{aligned}
$$

avec $c_{4}(N) \geq 2^{-N} N^{-2}$.

Remarque 4.3. Notons que la minoration de la conjecture 1.2, si elle est de nature purement géométrique, n'en est pas toujours meilleure que celle de la conjecture 1.1. Par exemple, dans $\mathbb{G}_{m}^{3}$, soit $X$ une courbe arbitraire dans le plan d'équation $x=a$ avec $a \in \mathbb{Q}^{\times} \backslash\{ \pm 1\}$. Alors $B_{X}=Z(x-a)$ et $U_{X}^{\mathbb{Q}}=\mathbb{G}_{m}^{3}$, d'où $\operatorname{deg}\left(B_{X}\right)=\operatorname{deg}\left(U_{X}^{\mathbb{Q}}\right)=1$ puis $\omega_{\mathbb{Q}}\left(X ; U_{X}^{\mathbb{Q}}\right)=1$ tandis que $\omega_{\overline{\mathbb{Q}}}\left(X ; B_{X}\right)=\operatorname{deg}(X)$ est arbitraire et donc

$$
\frac{\operatorname{deg}\left(U_{X}^{\mathbb{Q}}\right)}{\omega_{\mathbb{Q}}\left(X ; U_{X}^{\mathbb{Q}}\right)} \gg \frac{\operatorname{deg}\left(B_{X}\right)}{\omega_{\overline{\mathbb{Q}}}\left(X ; B_{X}\right)} .
$$

5. À propos du problème de Lehmer sur $\mathbb{k}(t)$. Soit $\mathbb{k}$ un corps algébriquement clos et $t$ une variable. Pour une extension finie $K$ de $\mathbb{k}(t)$ on considère un revêtement de la droite projective

$$
\pi: C_{K} \rightarrow \mathbb{P}^{1}(\mathbb{k})
$$

de degré $\operatorname{deg}(\pi)=[K: \mathbb{k}(t)]$ par une courbe projective lisse $C=C_{K}$, correspondant à l'extension de corps $\mathbb{k}(t) \hookrightarrow K$. Un élément $\xi \in K$ s'interprête ainsi comme une fonction rationnelle $\xi: C \rightarrow \mathbb{k}$, et pour une place $v \in C$ on note $\operatorname{ord}_{v}(\xi) \in \mathbb{Z}$ l'ordre d'annulation de $\xi$ en $v$. On a la « formule du produit », qui s'écrit additivement

$$
\sum_{v \in C} \operatorname{ord}_{v}(\xi)=0
$$


et la hauteur d'un point $\alpha=\left(\alpha_{0}: \cdots: \alpha_{N}\right) \in \mathbb{P}^{N}(K)$ est par définition

$$
h(\alpha):=\frac{1}{[K: \mathbb{k}(t)]} \sum_{v \in C} \max \left(-\operatorname{ord}_{v}\left(\alpha_{0}\right), \ldots,-\operatorname{ord}_{v}\left(\alpha_{N}\right)\right) .
$$

Cette formule est indépendante du choix des coordonnées projectives de $\alpha$ grâce à la formule du produit, et elle ne dépend pas non plus du choix du revêtement $\pi$.

Pour $\alpha \in \mathbb{P}^{N}(k(t))$ dont les coordonnées $\alpha_{j}$ sont des polynômes premiers entre eux, on a $h(\alpha)=\max _{j} \operatorname{deg}\left(\alpha_{j}\right)$. En outre, $h(\alpha)=0$ si et seulement si $\alpha$ est « constant» par rapport à $t$, c'est-à-dire si et seulement si $\alpha \in \mathbb{P}^{N}(\mathbb{k})$ : on le vérifie en supposant par exemple $\alpha_{0} \neq 0$, alors $h(\alpha)=0$ si et seulement si $\operatorname{ord}_{v}\left(\alpha_{j} / \alpha_{0}\right) \geq 0$ pour $j=1, \ldots, N$ et tout $v \in C$, ce qui équivaut à ce que $\alpha_{j} / \alpha_{0} \in \mathbb{k}$. Ainsi, les éléments de $\mathbb{k}$ jouent dans le cas fonctionnel le rôle des racines de l'unité dans le cas arithmétique.

L'application $\alpha: C \rightarrow \mathbb{P}^{N}(\mathbb{k})$ s'étend en une section globale $C \rightarrow \mathbb{P}^{N}(\mathbb{k})$ car $C$ est supposée lisse. Notons $X_{\alpha} \subset C \times \mathbb{P}^{N}(\mathbb{k})$ son graphe; on a [HS00, $\S \mathrm{B} .10]$

$$
h(\alpha)=\frac{1}{[K: \mathbb{k}(t)]} \operatorname{Card}\left(X_{\alpha} \cap p_{2}^{-1}(E)\right),
$$

où $p_{2}$ désigne la projection $C \times \mathbb{P}^{N}(\mathbb{k}) \rightarrow \mathbb{P}^{N}(\mathbb{k})$ et $E$ est un hyperplan générique de $\mathbb{P}^{N}(\mathbb{k})$.

Maintenant considérons le cas d'une variété $X \subset \mathbb{P}^{N}(\overline{\mathbb{k}(t)})$ de dimension quelconque $d \geq 0$, définie sur $K$. Considérons la variété $X \subset C \times \mathbb{P}^{N}(\mathbb{k})$ de dimension $d+1$ dont la fibre générique sur $C$ est égale à $X$. Soit $C \hookrightarrow \mathbb{P}^{M}(\mathbb{k})$ une immersion de $C$ dans un espace projectif. Soient

$$
p_{1}: C \times \mathbb{P}^{N}(\mathbb{k}) \rightarrow C \subset \mathbb{P}^{M}(\mathbb{k}), \quad p_{2}: C \times \mathbb{P}^{N}(\mathbb{k}) \rightarrow \mathbb{P}^{N}(\mathbb{k})
$$

les deux projections naturelles, et pour $d_{1}, d_{2} \geq 0$ tels que $d_{1}+d_{2}=d+1$ considérons le multidegré

$$
\operatorname{deg}_{\left(d_{1}, d_{2}\right)}(\mathcal{X}):=\operatorname{Card}\left(\mathcal{X} \cap p_{1}^{-1}\left(E_{1}\right) \cap p_{2}^{-1}\left(E_{2}\right)\right)
$$

où $E_{1} \subset \mathbb{P}^{M}$ et $E_{2} \subset \mathbb{P}^{N}$ désignent des espaces linéaires génériques de codimension $d_{1}$ et $d_{2}$, respectivement. La hauteur de la variété $X$ est alors par définition

$$
h(X):=\frac{1}{[K: \mathbb{k}(t)]} \operatorname{deg}_{(0, d+1)}(X) .
$$

On peut vérifier que ceci ne dépend pas des choix effectués, et qu'elle coïncide avec la définition précédente dans le cas des points. On a $h(X) \geq 0$ et de fait $h(X)=0$ si et seulement si $p_{2}(X)$ est de dimension $d$, ce qui équivaut à ce que $X$ soit définie sur $\mathbb{k}$. Pour les autres multidegrés, on a

$$
\operatorname{deg}_{\left(d_{1}, d_{2}\right)}(X)= \begin{cases}{[K: \mathbb{k}(t)] \operatorname{deg}(X)} & \text { pour } d_{1}=1, d_{2}=d \\ 0 & \text { pour } d_{1} \geq 2\end{cases}
$$


La hauteur et le degré de $X$ peuvent s'interpréter en termes de formes résultantes de l'idéal bihomogène $I(X) \subset \mathbb{k}\left[w_{0}, \ldots, w_{M}, x_{0}, \ldots, x_{N}\right]$. Soient $W$ un groupe de $M+1$ variables et $V_{0}, \ldots, V_{d}$ des groupes de $N+1$ variables chacun et soit

$$
\text { rés } x \in \mathbb{k}\left[W, V_{0}, \ldots, V_{d}\right]
$$

la forme résultante de $I(\mathcal{X})$ d'indice $(1,0)$ et $(0,1), \ldots,(0,1)(d+1$ fois $)$ (voir [Rém01, §3] ou encore [PS04, §I.2] pour la définition et propriétés de base des formes résultantes). C'est un polynôme multihomogène dont le degré par rapport à chaque groupe de variables est [Rém01, Prop. 3.4 et 2.11]

$$
\begin{aligned}
& \operatorname{deg}_{W}(\operatorname{rés} X)=\operatorname{deg}_{(0, d+1)}(X)=[K: \mathbb{k}(t)] h(X), \\
& \operatorname{deg}_{V_{i}}(\operatorname{rés} X)=\operatorname{deg}_{(1, d)}(X)=[K: \mathbb{k}(t)] \operatorname{deg}(X), \quad \text { pour } i=1, \ldots, d .
\end{aligned}
$$

Comme conséquence de cette interprétation et de [Rém01, Lem. 2.11] on peut démontrer le théorème de Bézout fonctionnel : si

$$
f \in \mathbb{k}\left[w_{0}, \ldots, w_{M}, x_{0}, \ldots, x_{N}\right] \backslash I(\mathcal{X})
$$

est une forme bihomogène, alors

$$
h(X \cdot \operatorname{div}(f))=\operatorname{deg}_{\mathbf{x}}(f) h(X)+\operatorname{deg}_{\mathbf{w}}(f) \operatorname{deg}(X) .
$$

Similairement, on obtient une formule de Hilbert-Samuel pour la dimension de l'espace des formes de bidegré $(\eta, \delta)$ modulo $I(X)$ [Rém01, Thm. 2.10] :

$$
\begin{aligned}
\operatorname{dim}_{\mathbb{k}}\left(\mathbb{k}[X]_{(\eta, \delta)}\right) \\
=\frac{\operatorname{deg}_{(0, d+1)}(X)}{(d+1) !} \delta^{d+1}+\frac{\operatorname{deg}_{(1, d)}(X)}{d !} \eta \delta^{d}+O\left((\eta+\delta) \delta^{d-1}\right) \\
=[K: \mathbb{k}(t)]\left(\frac{h(X)}{(d+1) !} \delta^{d+1}+\frac{\operatorname{deg}(X)}{d !} \eta \delta^{d}\right)(1+o(1))
\end{aligned}
$$

pour $\eta, \delta \rightarrow \infty$.

Par ailleurs, le minimum essentiel de $X$ est naturellement défini par

$$
\mu^{\text {ess }}(X):=\inf \{\theta:\{\xi \in X: h(\xi) \leq \theta\} \text { est Zariski dense }\} .
$$

On pose $U_{X} \subset \mathbb{P}^{N}(\overline{\mathbb{k}(t)})$ la plus petite variété définie sur $\mathbb{k}$ et contenant $X$. Le résultat principal de ce paragraphe est l'analogue fonctionnel suivant de la conjecture 1.1 :

Proposition 5.1. Soit $X \subset \mathbb{P}^{N}(\overline{\mathbb{k}(t)})$ une variété de dimension $d \geq 0$. Alors

$$
(d+1) \mu^{\mathrm{ess}}(X) \geq \frac{h(X)}{\operatorname{deg}(X)} \geq \frac{\operatorname{deg}\left(U_{X}\right)}{\omega_{\mathbb{k}(t)}\left(X ; U_{X}\right)} .
$$

Démonstration. La première inégalité résulte de la proposition 5.2 cidessous. Pour la seconde, on remarque $U_{X}=p_{2}(X) \subset \mathbb{P}^{N}(\mathbb{k})$. Cette variété est donc égale à $X$ si $X$ est définie sur $\mathbb{k}$, et de dimension $d+1$ sinon. 
Si $X=U_{X}$ on a $\omega_{\mathbb{k}(t)}\left(X ; U_{X}\right)=\infty$ et le résultat est clair, sinon $X$ est de codimension 1 dans $U_{X}$. On a clairement

$$
\operatorname{deg}_{(0, d+1)}(X)=\operatorname{deg}\left(U_{X}\right) \operatorname{deg}\left(p_{2} \mid x\right) \geq \operatorname{deg}\left(U_{X}\right) .
$$

D'un autre côté, le diviseur minimal de $U_{X}$ défini sur $\mathbb{k}(t)$ et contenant $X$ est $\bigcup_{\sigma} \sigma(X)$ où $\sigma$ parcourt les $\mathbb{k}(t)$-immersions du corps de définition $K$ de $X$ dans la clôture algébrique $\overline{\mathbb{k}(t)}$. Le degré de ce diviseur est $[K: \mathbb{k}(t)] \operatorname{deg}(X)$ et donc $\omega_{\mathbb{k}(t)}\left(X ; U_{X}\right)=[K: \mathbb{k}(t)] \operatorname{deg}(X)$. On en conclut

$$
\frac{h(X)}{\operatorname{deg}(X)}=\frac{\operatorname{deg}_{(0, d+1)}(X)}{\operatorname{deg}_{(1, d)}(X)} \geq \frac{\operatorname{deg}\left(U_{X}\right)}{[K: \mathbb{k}(t)] \operatorname{deg}(X)}=\frac{\operatorname{deg}\left(U_{X}\right)}{\omega_{\mathbb{k}(t)}\left(X ; U_{X}\right)} .
$$

Il ne reste qu'à démontrer l'analogue fonctionnel suivant du théorème des minimums algébriques successifs. On reprend pour cela en partie les idées de [DP98, Thm. 3.1].

Proposition 5.2. Soit $X \subset \mathbb{P}^{N}(\overline{\mathbb{k}(t)})$ une variété de dimension $d \geq 0$. Alors

$$
\mu^{\mathrm{ess}}(X) \leq \frac{h(X)}{\operatorname{deg}(X)} \leq(d+1) \mu^{\mathrm{ess}}(X) .
$$

Démonstration. Pour l'inégalité de gauche, il suffit de démontrer que pour tout diviseur $Z$ de $X$ il y a des points dans $X \backslash Z$ de hauteur $\leq$ $h(X) / \operatorname{deg}(X)$. En intersectant $X$ avec $d$ formes linéaires génériques à coefficients dans $\mathbb{k}$ le cycle intersection est de dimension 0 , de degré $\operatorname{deg}(X)$ et à support dans $X \backslash Z$. Par le théorème de Bézout (formule (10) ci-dessus) la hauteur de ce cycle est $h(X)$, et donc son support contient au moins un point de hauteur $\leq h(X) / \operatorname{deg}(X)$.

Pour l'inégalité de droite, on commence par supposer sans perte de généralité que $X$ est définie sur $\mathbb{k}(t)$. Soit $I(X)$ l'idéal de définition de $X$ dans $\mathbb{k}(t)\left[x_{0}, \ldots, x_{N}\right]$ et $\varepsilon>0$. Il existe une base de l'espace dual $I(X)_{\delta}^{\vee}$ de la forme

$$
b_{i}=\left(\xi_{i}^{\alpha}\right)_{\alpha \in \mathbb{N}^{N+1},|\alpha|=\delta}, \quad i=1, \ldots, L:=\operatorname{dim}_{\mathbb{k}(t)}\left(\mathbb{k}(t)[X]_{\delta}\right),
$$

avec $\xi_{i} \in X(\overline{\mathbb{k}(t)})$ et $h\left(\xi_{i}\right) \leq \mu^{\text {ess }}(X)+\varepsilon$, par définition du minimum essentiel. On en déduit à l'aide de la formule de Brill-Gordan que

$$
h\left(I(X)_{\delta}\right)=h\left(I(X) \frac{\perp}{\delta}\right) \leq \sum_{i=1}^{L} h\left(b_{i}\right)=\delta \sum_{i=0}^{L} h\left(\xi_{i}\right) \leq \delta L\left(\mu^{\mathrm{ess}}(X)+\varepsilon\right) .
$$

Par ailleurs on a, en posant $w=\left(w_{0}, w_{1}\right)$ et $x=\left(x_{0}, \ldots, x_{N}\right)$,

$$
\begin{aligned}
\operatorname{dim}_{\mathbb{k}}\left(\mathbb{k}[X]_{(\eta, \delta)}\right) & =\operatorname{dim}_{\mathbb{k}}\left(\mathbb{k}[w, x]_{(\eta, \delta)}\right)-\operatorname{dim}_{\mathbb{k}}\left(I(X)_{(\eta, \delta)}\right) \\
& =(\eta+1)\left(\begin{array}{c}
\delta+N \\
N
\end{array}\right)-\operatorname{dim}_{\mathbb{k}}\left(I(X)_{(\eta, \delta)}\right) .
\end{aligned}
$$


Notons $J:=\operatorname{dim}_{\mathbb{k}(t)}\left(I(X)_{\delta}\right)=\left(\begin{array}{c}\delta+N \\ N\end{array}\right)-L$. D'après [Thu95, Cor. 2] (voir aussi [Mah41, p. 489]) il existe une base $c_{1}, \ldots, c_{J}$ du $\mathbb{k}(t)$-espace $I(X)_{\delta}$ satisfaisant $\sum_{j=1}^{J} h\left(c_{j}\right)=h\left(I(X)_{\delta}\right)$. Mais alors les éléments

$$
t^{l} c_{j}, \quad j=1, \ldots, J, l=0, \ldots, \eta-h\left(c_{j}\right),
$$

sont linéairement indépendants sur $\mathbb{k}$ dans $I(X)_{(\eta, \delta)}$ qui est donc de dimension au moins $(\eta+1) J-\sum_{j=1}^{J} h\left(c_{j}\right) \geq(\eta+1) J-h\left(I(X)_{\delta}\right)$. En reportant dans (13) on obtient

$$
\begin{aligned}
\operatorname{dim}_{\mathbb{k}}\left(\mathbb{k}[X]_{(\eta, \delta)}\right) & \leq(\eta+1)\left(\begin{array}{c}
\delta+N \\
N
\end{array}\right)-(\eta+1) J+h\left(I(X)_{\delta}\right) \\
& \leq(\eta+1) L+h\left(I(X)_{\delta}\right) .
\end{aligned}
$$

En réunissant (11), (12) et (14) il vient avec l'expression asymptotique de $L$ comme fonction de Hilbert :

$$
\begin{aligned}
& \left(\frac{h(X)}{(d+1) !} \delta^{d+1}+\frac{\operatorname{deg}(X)}{d !} \eta \delta^{d}\right)(1+o(1)) \\
& \quad \leq \frac{\operatorname{deg}(X)}{d !} \delta^{d}\left(\eta+1+\delta\left(\mu^{\mathrm{ess}}(X)+\varepsilon\right)\right)(1+o(1))
\end{aligned}
$$

et enfin, en divisant par $\delta^{d+1}$ et en faisant tendre $\eta$ et $\delta$ vers l'infini de sorte que $\eta / \delta$ reste borné,

$$
\frac{h(X)}{(d+1) !} \leq \frac{\operatorname{deg}(X)}{d !}\left(\mu^{\operatorname{ess}}(X)+\varepsilon\right),
$$

puis le résultat voulu lorsque $\varepsilon$ tend vers 0 .

\section{Références}

[ADa01] F. Amoroso et S. David, Densité des points à coordonnées multiplicativement indépendantes, Ramanujan J. 5 (2001), 237-246.

[ADa03] -, -, Minoration de la hauteur normalisée dans un tore, J. Inst. Math. Jussieu 2 (2003), 335-381.

[ADe06] F. Amoroso et E. Delsinne, Une minoration relative explicite pour la hauteur dans une extension d'une extension abélienne, tapuscript, $23 \mathrm{pp}$.

[ADv00] F. Amoroso and R. Dvornicich, A lower bound for the height in abelian extensions, J. Number Theory 80 (2000), 260-272.

[AN07] F. Amoroso and F. Nuccio, Algebraic numbers of small Weil's height in CMfields: on a theorem of Schinzel, J. Number Theory 122 (2007), 247-260.

[AZ00] F. Amoroso and U. Zannier, A relative Dobrowolski lower bound over abelian extensions, Ann. Scuola Norm. Sup. Pisa Cl. Sci. (4) 29 (2000), 711-727.

[Bern75] D. N. Bernshtein, The number of roots of a system of equations, Funktsional. Anal. i Prilozhen. 9 (1975), no. 3, 1-4 (en russe).

[Ber95] D. Bertrand, Minimal heights and polarizations on group varieties, Duke Math. J. 80 (1995), 223-250. 
[Ber97] D. Bertrand, Duality on tori and multiplicative dependence relations, J. Austral. Math. Soc. 62 (1997), 198-216.

[Cas71] J. W. S. Cassels, An Introduction to the Geometry of Numbers, Grundlehren Math. Wiss. 99, Springer, 1971.

[Cha89] M. Chardin, Une majoration de la fonction de Hilbert et ses conséquences pour l'interpolation algébrique, Bull. Soc. Math. France 117 (1989), 305-318.

[Dav03] S. David, On the height of subvarieties of groups varieties, tapuscrit, $61 \mathrm{pp}$.

[DP98] S. David et P. Philippon, Minorations des hauteurs normalisées des sousvariétés de variétés abéliennes, dans : Number Theory (Tiruchirapalli, 1996), Contemp. Math. 210, Amer. Math. Soc., 1998, 333-364.

[DP99] - - - Minorations des hauteurs normalisées des sous-variétés des tores, Ann. Scuola Norm. Sup. Pisa Cl. Sci. (4) 28 (1999), 489-543.

[Dob79] E. Dobrowolski, On a question of Lehmer and the number of irreducible factors of a polynomial, Acta Arith. 34 (1979), 391-401.

[ES96] D. Eisenbud and B. Sturmfels, Binomial ideals, Duke Math. J. 84 (1996), 1-45.

[Ewa96] G. Ewald, Combinatorial Convexity and Algebraic Geometry, Springer, 1996.

[HS00] M. Hindry and J. Silverman, Diophantine Geometry. An Introduction, Springer, 2000.

[Mah41] K. Mahler, An analogue to Minkowski's geometry of numbers in a field of series, Ann. of Math. (2) 42 (1941), 488-522.

[PS04] P. Philippon et M. Sombra, Hauteur normalisée des variétés toriques projectives, J. Inst. Math. Jussieu, à paraître; http://fr.arxiv.org/abs/math.NT /0406476, $38 \mathrm{pp}$.

[PS05] - - - Quelques aspects diophantiens des variétés toriques projectives, dans : H. P. Schlickewei et R. Tichy (eds.), volume en l'honneur de Wolfgang Schmidt, Dev. Math., Springer, à paraître; http://fr.arxiv.org/abs/math.NT/0411084, $40 \mathrm{pp}$.

[Rém01] G. Rémond, Élimination multihomogène, chapitre 5 de : Introduction to Algebraic Independence Theory, Lecture Notes in Math. 1752, Springer, 2001, 53-81.

[Sch73] A. Schinzel, On the product of the conjugates outside the unit circle of an algebraic number, Acta Arith. 24 (1973), 385-399; Addendum, ibid. 26 (1975), 329-331.

[Thu95] J. Thunder, Siegel's lemma for function fields, Michigan Math. J. 42 (1995), $147-162$.

Projet Géométrie et Dynamique

Departament d'Àlgebra i Geometria

Institut de Mathématiques de Jussieu (U.M.R. 7586)

Universitat de Barcelona

Case 7512

Gran Via 585

2 place Jussieu

75251 Paris Cedex 05, France

08007 Barcelona, Espagne

E-mail: pph@math.jussieu.fr

E-mail: sombra@ub.edu

http://www.math.jussieu.fr/ pph/

http://atlas.mat.ub.es/personals/sombra/ 